\title{
Combination of Sorbents and Modification of Its Constituents to Enhance the Mopping Ability of Chemically Modified and Unmodified Biological Wastes on Crude Oil and Its Lower Fractions
}

\author{
John Kanayochukwu Nduka \\ Environmental Chemistry and Toxicology Research Unit, Pure and Industrial Chemistry Department, \\ Nnamdi Azikiwe University, Awk, Nigeria \\ Email: johnnduka2000@yahoo.co.uk
}

Received April 2, 2012; revised May 19, 2012; accepted May 24, 2012

\begin{abstract}
Protein wastes (feathers, goat hair) and cellulosic wastes (corn cob, coconut husks) were collected, washed with detergent solution, thoroughly rinsed and sun dried for 2 days before drying in an oven and then ground, half of ground material was carbonized at a maximum temperature of $500^{\circ} \mathrm{C}$ after mixing with $\mathrm{H}_{2} \mathrm{SO}_{4}$. The carbonized parts were pulverized; both carbonized and uncarbonized sorbents were sieved into two particle sizes of $325 \mu \mathrm{m}$ and $625 \mu \mathrm{m}$ using mechanical sieve.1.5 g protein wastes and cellulosic wastes were each used to mop up spilled crude oil, diesel, kerosene and petrol (separately before combining) by encasing them in a sac like boom of $2 \mathrm{~cm} \mathrm{x} 2 \mathrm{~cm} \mathrm{x} 1 \mathrm{~cm}$ dimension so as to determine the efficacy of waste sorbents in cleaning hydrocarbon spills. Results of Tables 1(a)-4(a), 1(b)-4(b) and 5-8 show that the sorbents mopped, desorbed and retained an average of more than $500 \%, 350 \%$ and $300 \%$ of their weight of the hydrocarbon sorbates within average of 90 mins contact time, the volume of sorbates mopped up increased significantly when equal weight of activated and unactivated sorbents were combined, the results of equal combination of activated and unactivated sorbents at each particle size and contact time were compared with that of calculated values, high percentage retention observed were a function of mechanism of absorption/adsorption. Each sorbent have a distinct feature that enhances its mopping ability. Large volumes of the hydrocarbon liquids were recovered by mere pressing, the sorbates were mopped up in the order; crude oil $>$ diesel $>$ kerosene $>$ petrol. Protein sorbents with oleophilic and aqua phobic properties absorbed more of all the hydrocarbon liquids than cellulosic sorbents at any particle size and contact, the later tend to be more abundant and therefore cost effective; it was observed that both carbonized and uncarbonized sorbents are good hydrocarbon mops and therefore good alternative to synthetic polyurethane foam already in use. Combination, particle size, activation of sorbents, contact time, viscosity and chain length of hydrocarbon determined the amount of sorbates absorbed/adsorbed, recovered or retained. The residual leachable oil (kerosene) in the sorbents were below 5\% and does not constitute serious environmental menace when left in an open dump to decay being biodegradable waste, but a brighter application is that the waste sorbent can be used in making logs as alternative to fire wood or in making particle board for furniture.
\end{abstract}

Keywords: Sorbent-Sorbate System; Sorbents Combination; Experimental and Calculated Value; Adsorption/Absorption; Contact Time; Re-Use

\section{Introduction}

Crude oil production (exploration and exploitation) is Nigeria's largest and most important industrial sector. This came to be after the most reliable and ubiquitous sustainer of the economy-agriculture has been relegated to the background [1]. Nigeria's Niger delta (situated on the continental margin of the Gulf of Guinea in the equatorial West Africa between latitude $3^{\circ}$ and $6^{\circ}$ North and longitude $5^{\circ}$ and $8^{\circ}$ East) can be regarded as crude oil basin. Its crude oil reserve is estimated at about more than 30 billion barrels of crude oil and 260 trillion cubic feet of natural gas. The country's Niger delta ranks amongst the world's most prolific petroleum tertiary deltas that together accounts for about $5 \%$ of the world's oil and gas reserves and for about $2.5 \%$ of the present basin area on earth [2]. Man's attempt to economically utilize the crude oil reserves entails several operations such as; exploration/drilling operation (drilling and work-over activities), production operations (production facilities 
include producing wells, flow stations, gas plants, gas and water injection stations and numerous pipelines that connect these facilities), terminal operations (which involves filling of tanks, barges, vessels, dehydration of crude, crude storage, effluent water, disposal and loading of tankers), engineering operations (this include dredging, flow-line replacement, flow-station upgrade), sabotage/ theft (vandalization of manifolds, pipelines, delivery lines, cutting or removal of pipelines), etc. [3]. The above activities involve a certain amount of alteration of environment in a conspicuously negative manner. Vegetation is cleared to make way for seismic lines, sites for drilling rigs are leveled and roads are built [1]. The inevitable effect is the millions of barrels of crude oil and its fractions that are spilled in the Niger delta region. The crude oil from which myriad of fractions can be obtained is a dark, thick, odoriferous liquid composed of about 95\% hydrocarbons such as alkanes, cycloalkanes and aromatics [4]. According to Nigeria's Department of Petroleum Resources (DPR); magnitude of oil spillage can be classified into minor spill (less than 25 barrels discharged on inland water or coastal/offshore water; medium spill (25 to 250 barrels discharged on land or coastal/offshore water; major spill (greater than 250 barrels discharged on inland water or greater than 2500 barrels discharged on land, coastal/offshore waters [3]. Remediating and cleanup procedures must take into account of the magnitude of the spill. Previous works that relate with highly fibrillated fibrous materials for cleaning failing protein water [5]; micro fibrous sorbents on the basis of thermoplastic polymers [6] and wool fibrous materials of high surface area for cleaning of oil spills in the Persian Gulf in 1990 [7] are available. Also highly effective sorbents on the basis of fibrous waste from paper production [8], sorption properties of lignin-derivatised fibrous composites for the remediating oil polluted receiving waters [9] and the use of lignin-containing waste for cleaning water [10] have been reported. Combination of activated (modified) and unactivated (unmodified) sorbents to enhance its oil sorption capacity has not been studied. The aim of this work is to combine sorbents of different treatment but the same particle size and compare its mopping capability with calculated value and also to see if the sorbents natural constituent and modification due to activation enhanced their oil mopping ability.

\section{Materials and Method}

Collection of sorbents and preparation; cellulosic sorbents (coco-nut husk (coir), corn-cob) were procured from a local market around Awka while protein sorbents (chicken feather, goat hair) were sourced from Awka main abattoir. They were thoroughly washed with detergent solution, sun dried for two days before drying in an oven at $150^{\circ} \mathrm{C}$ for $1 \mathrm{hr}$ and then ground. One half of each ground material was carbonized at a temperature of $300^{\circ} \mathrm{C}$ for 30 mins, cooled and activated with $1 \mathrm{MH}_{2} \mathrm{SO}_{4}$ and further heated at a temperature of $500^{\circ} \mathrm{C}$ for complete carbonization. The carbonized materials were again pulverized. Both carbonized and uncarbonized materials were sieved into two particle sizes of $325 \mu \mathrm{m}$ and 625 $\mu \mathrm{m}$ using mechanical sieve. Nylon 66 fabric whose adsorption profiles had been predetermined and found to be adequate was used for the construction of pillow cases/ bags $(2 \mathrm{~cm} \times 2 \mathrm{~cm} \times 1 \mathrm{~cm})$ for the sorbents [11]

The sorbates: Crude oil

Assay: [C (82\%), H (15\%), N (0.02\%), O (0.5\%)], Diesel $\left(\mathrm{C}_{15}-\mathrm{C}_{20}\right)$; Kerosene $\left(\mathrm{C}_{9}-\mathrm{C}_{15}\right)$ and Petrol $\left(\mathrm{C}_{5}-\mathrm{C}_{9}\right)$ was procured from Port Harcourt Refinery, Nigeria.

\section{Methods}

Exactly equal combinations $(1.5 \mathrm{~g})$ of both carbonized and uncarbonized sorbents $(0.75 \mathrm{~g}$ of carbonized and $0.75 \mathrm{~g}$ of uncarbonized feather; $0.75 \mathrm{~g}$ of carbonized and $0.75 \mathrm{~g}$ of uncarbonized goat hair; $0.75 \mathrm{~g}$ carbonized and $0.75 \mathrm{~g}$ of uncarbonized coco-nut husk (coir); $0.75 \mathrm{~g}$ carbonized corn-cob and $0.75 \mathrm{~g}$ of uncarbonized corn cob) were each separately encased in the pillow cases/bags, stitched and weight noted. Four (4) pillow cases/bags each for a given combination of sorbents (equal grammes of both carbonized and uncarbonized) for a given particle size $(325 \mu \mathrm{m}$ and $625 \mu \mathrm{m})$ were introduced into the test liquid (sorbates) and completely immersed and withdrawn one after the other at 30, 60, 90 and 120 mins intervals. After $1.5 \mathrm{~g}$ of sorbent was used singly without combination. Each pillow/bag was hung in air for $5 \mathrm{mins}$ to drain off unabsorbed surface liquid. The quantity of liquid actually adsorbed by the sorbents was determined by weighing. The amount of sorbate recovered was obtained by pressing the pillow at room temperature on a carver hydraulic press model M, Ser. No. 12000-137 at a pressure of 25 tonnes, for 3mins for a sorbent-sorbate system. Average of three (3) operations was taken for absorption/adsorption and recovery for a particular test, the percentage absorption/adsorption, recovery and retention were calculated. They were repeated up to four (4) times. The concentration and eventual calculation of percentage leachable oil was determined by soaking $3 \mathrm{~g}$ of the sorbents with kerosene (having previously determined that of crude oil and diesel) for $1 \mathrm{hr}$ after which the oil was squeezed out, the squeezed sorbents was soaked in $10 \mathrm{ml}$ of warm xylene for $24 \mathrm{hrs}$ to extract the residual oil from the sorbents. After filtration, the xylene layer was dried and the absorbance of the extract read on a UVvisible spectrophotometer at a wavelength of $420 \mathrm{~nm}$, the result compared with a standard. This procedure was repeated at 48, 72 and $96 \mathrm{hrs}$ interval [11]. Calculated 
values were gotten by using $1.5 \mathrm{~g}$ of each sorbent (carbonized and uncarbonized) separately to mop each sorbate as reported in our previous work [12], their average results reported as calculated value (i.e. percentage absorption/adsorption of carbonized sorbent plus percentage absorption/adsorption of uncarbonized sorbent of the same particle size divided by two (2) is given as calculated percentage absorption; percentage recovery of carbonized sorbent plus percentage recovery of uncarbonized sorbent of the same particle size divided by two (2) is given as calculated percentage recovery; percentage retention of carbonized sorbents plus percentage retention of uncarbonized sorbents of the same particle size divided by two (2) is given as calculated percentage retention (Tables 1(b)-4(b)),

\section{Calculations:}

$\%$ absorption/adsorption

$=\frac{\text { Final weight }- \text { initial weight } \times 100}{\text { Initial weight }}$

$\%$ recovery

$=\frac{\text { Final weight }- \text { weight after pressing } \times 100}{\text { Initial weight }}$

$\%$ retention $=\%$ absorption/adsorption $-\%$ recovery

Calculation for leachability test

$\%$ leachability $=100-\%$ retention

$$
\% \text { retention }=\frac{\mathrm{A}-\mathrm{B} \times 100}{\mathrm{~A}}
$$

where $\mathrm{A}=$ initial concentration of leachable oil (kerosene),

B = Concentration of leachable oil after 24, 48, 72 and 96hrs.

\section{Determination of protein, lignin and cellulose}

The total nitrogen of the proteinous sorbents (feather and goat hair) was determined by the Kjeldahl method and then converted to crude protein [13]. The plant sorbents (coco-nut husk (coir) and corn cob) were analyzed for $\alpha$-cellulose and acid insoluble (klason) lignin; $\alpha$-cellulose was determined using a colorimetric method with the anthrone reagent. $0.3 \mathrm{~g}$ (dry weight) ground $(0.5 \mathrm{~mm})$ samples were treated and boiled $\left(\right.$ at $\left.100^{\circ} \mathrm{C}\right)$ with a mixture of nitric/acetic acid $(1.8 ; \mathrm{V} / \mathrm{V})$ for $1 \mathrm{hr}$ to remove lignin, hemicelluloses and xylosans after successive centrifugations, and diluted with $67 \% \mathrm{H}_{2} \mathrm{SO}_{4}(\mathrm{~V} / \mathrm{V})$. Cellulose was then determined at $620 \mathrm{~nm}$ using cold antrone reagent [14]. The method is suitable for analyzing a large number of samples and has been used to determine cellulose in other plant materials [15].

\section{Determination of heavy metal}

The sorbents were ashed and digested using aqua regia. The filtrates after digestion were analyzed for $\mathrm{Cr}, \mathrm{Fe}, \mathrm{Ni}$ and $\mathrm{Mn}$ using atomic absorption spectrophotometry
(AAS), 200A at their respective wavelengths $(358,247$, 231 and 278) $\mathrm{nm}$.

\section{Results}

Table 1(a): \% absorption/recovery profiles of sorbates by equal combination ( $0.75 \mathrm{~g}$ each) of carbonized and uncarbonized feather at each particles size and contact time. Using $325 \mu \mathrm{m}$, crude oil was absorbed and recovered highest at 120 mins, higher percentage absorption/ recovery continues across the table even up to $4^{\text {th }}$ use, highest recovery was at both 90 and 120 mins. Diesel was absorbed/recovered highest at 120 mins contact time at $1^{\text {st }}$ use, the same at $2^{\text {nd }}$ use but was absorbed highest at both 30 and 120 mins time at $3^{\text {rd }}$ use while highest recovery was at 90 and 120 mins. Kerosene was absorbed/recovered highest at 120 mins at $1^{\text {st }}$ use, the same trend was observed at $2^{\text {nd }}$ and $3^{\text {rd }}$ use. Petrol was absorbed and for first application, $2^{\text {nd }}$ use had highest absorption at both 90 and 120 mins but had highest recovery at 120 mins. Using $625 \mu \mathrm{m}$, crude oil was absorbed/ recovered highest at $120 \mathrm{mins}$ from $1^{\text {st }}$ to $2^{\text {nd }}$ use but highest recovery at $3^{\text {rd }}$ use occurred at 90 mins contact time Diesel was absorbed highest at 120 mins from $1^{\text {st }}$ to $4^{\text {th }}$ use, the same for recovery except that highest recovery for $3^{\text {rd }}$ use occurred at 60 and 90 mins. Kerosene had highest absorption/recovery at 120 mins for both $1^{\text {st }}$ and $2^{\text {nd }}$ use; petrol was absorbed highest at 90 mins and recovered highest at $60 \mathrm{mins}$ for $1^{\text {st }}$ use. Generally, minor variation do exists.

Table 1(b): Calculated \% absorption/recovery profiles of sorbates by equal combination of carbonized and uncarbonized feather at different particles size and contact time. Using $325 \mu \mathrm{m}$ particles size, crude oil was absorbed/recovered highest at $1^{\text {st }}$ use at 120 mins, $2^{\text {nd }}$ use was at 60 mins contact time, highest for $3^{\text {rd }}$ and $4^{\text {th }}$ use was at 90 mins but recovery was at, 90 and 30 mins respectively. Diesel was absorbed/recovered most at 120 mins time during $1^{\text {st }}$ and $2^{\text {nd }}$ application; kerosene was absorbed highest at 120 mins but was recovered most at 30 mins contact time, during $1^{\text {st }}$ application, highest absorption/recovery was at 120 mins for $2^{\text {nd }}$ to $4^{\text {th }}$ use. Petrol was absorbed more at 120 mins during $1^{\text {st }}$ use, highest absorption from $2^{\text {nd }}$ to $4^{\text {th }}$ occurred at 60 mins contact time. Using $625 \mu \mathrm{m}$, crude oil was absorbed and recovered highest at 120 mins contact time from $1^{\text {st }}$ to $4^{\text {th }}$ use. Diesel was absorbed most from 60 up to 120 mins at $1^{\text {st }}$ use while recovery was highest at $60 \mathrm{mins}, 2^{\text {nd }}$ application has highest absorption and recovery at 60 mins and 120 mins respectively, Kerosene was absorbed/recovered highest at 120 mins contact time from $1^{\text {st }}$ to $4^{\text {th }}$ application, the same trend was observed for petrol though minor variation existed.

Table 2(a): Percentage absorption/recovery profiles 
Table 1. (a) Percentage absorption/recovery profiles of sorbates by equal combination of carbonized and uncarbonized feather at different particles size and contact time; (b) Calculated percentage absorption/recovery profiles of sorbates by equal combination of carbonized and uncarbonized feather at different particles size and contact time.

(a)

\begin{tabular}{|c|c|c|c|c|c|c|c|}
\hline \multicolumn{8}{|c|}{$\%$ Absorption/Recovery } \\
\hline S/No & Particle Size & Contact time (min) & Sorbates & $\mathbf{1}^{\text {st }}$ Use & $2^{\text {nd }}$ Use & $3^{\text {rd }}$ Use & $4^{\text {th }}$ Use \\
\hline \multirow{4}{*}{1} & \multirow{4}{*}{$325 \mu \mathrm{m}$} & 30 & \multirow{4}{*}{ Crude oil } & $560(380)$ & $550(380)$ & $520(385)$ & $500(370)$ \\
\hline & & 60 & & $680(400)$ & $650(390)$ & $600(390)$ & $480(375)$ \\
\hline & & 90 & & $710(410)$ & $700(400)$ & $650(395)$ & $475(380)$ \\
\hline & & 120 & & $720(410)$ & $710(400)$ & $700(400)$ & $520(385)$ \\
\hline \multirow{4}{*}{2} & \multirow{4}{*}{$325 \mu \mathrm{m}$} & 30 & \multirow{4}{*}{ Diesel } & $530(380)$ & $510(280)$ & $500(280)$ & $420(285)$ \\
\hline & & 60 & & $560(385)$ & $520(285)$ & $505(275)$ & $410(280)$ \\
\hline & & 90 & & $580(390)$ & $510(280)$ & $495(270)$ & $425(280)$ \\
\hline & & 120 & & $600(400)$ & $550(295)$ & $500(290)$ & $450(290)$ \\
\hline \multirow{4}{*}{3} & \multirow{4}{*}{$325 \mu \mathrm{m}$} & 30 & \multirow{4}{*}{ Kerosene } & $465(300)$ & $430(280)$ & $400(280)$ & $380(200)$ \\
\hline & & 60 & & $470(290)$ & $470(290)$ & $430(290)$ & $400(250)$ \\
\hline & & 90 & & $485(295)$ & $465(295)$ & $440(265)$ & $420(260)$ \\
\hline & & 120 & & $495(350)$ & $470(300)$ & $450(300)$ & $435(265)$ \\
\hline \multirow{4}{*}{4} & \multirow{4}{*}{$325 \mu \mathrm{m}$} & 30 & \multirow{4}{*}{ Petrol } & $390(260)$ & $380(200)$ & $380(185)$ & $360(160)$ \\
\hline & & 60 & & $420(270)$ & $396(210)$ & $390(200)$ & $355(158)$ \\
\hline & & 90 & & $425(280)$ & $400(200)$ & $396(186)$ & $370(165)$ \\
\hline & & 120 & & $440(290)$ & $400(210)$ & 394 (192) & $380(170)$ \\
\hline \multirow{4}{*}{5} & \multirow{4}{*}{$625 \mu \mathrm{m}$} & 30 & \multirow{4}{*}{ Crude Oil } & $505(360)$ & $490(360)$ & $415(200)$ & 405 (210) \\
\hline & & 60 & & $535(370)$ & $486(370$ & $430(190)$ & $400(205)$ \\
\hline & & 90 & & $560(365)$ & $490(365)$ & $420(195)$ & $410(200)$ \\
\hline & & 120 & & $670(390)$ & $500(380)$ & $455(290)$ & $435(210)$ \\
\hline \multirow{4}{*}{6} & \multirow{4}{*}{$625 \mu \mathrm{m}$} & 30 & \multirow{4}{*}{ Diesel } & $410(280)$ & $400(270)$ & $380(190)$ & $320(160)$ \\
\hline & & 60 & & $440(275)$ & $395(255)$ & $400(200)$ & $360(180)$ \\
\hline & & 90 & & $430(285)$ & $420(265)$ & $405(200)$ & 385 (175) \\
\hline & & 120 & & $460(296)$ & $425(280)$ & 410 (195) & $396(190)$ \\
\hline \multirow{4}{*}{7} & \multirow{4}{*}{$625 \mu \mathrm{m}$} & 30 & \multirow{4}{*}{ Kerosene } & $380(200)$ & 385 (195) & $370(180)$ & $360(170)$ \\
\hline & & 60 & & $425(210)$ & $400(200)$ & $390(180)$ & $385(180)$ \\
\hline & & 90 & & $415(240)$ & $410(185)$ & $385(175)$ & $375(170)$ \\
\hline & & 120 & & $430(260)$ & $430(220)$ & $395(190)$ & $380(180)$ \\
\hline \multirow{4}{*}{8} & \multirow{4}{*}{$625 \mu \mathrm{m}$} & 30 & \multirow{4}{*}{ Petrol } & $365(180)$ & $360(175)$ & $350(160)$ & $310(160)$ \\
\hline & & 60 & & $380(200)$ & $370(178)$ & $345(155)$ & $330(158)$ \\
\hline & & 90 & & $395(190)$ & $380(180)$ & $360(160)$ & $340(162)$ \\
\hline & & 120 & & $390(185)$ & $385(185)$ & $375(170)$ & $355(165)$ \\
\hline
\end{tabular}

[\% Recovery in parenthesis]. 
(b)

\begin{tabular}{|c|c|c|c|c|c|c|c|}
\hline \multicolumn{8}{|c|}{$\%$ Absorption/Recovery } \\
\hline S/No & Particle size & Contact time (min) & Sorbates & $\mathbf{1}^{\text {st }}$ Use & $2^{\text {nd }}$ Use & $3^{\text {rd }}$ Use & $4^{\text {th }}$ Use \\
\hline \multirow{4}{*}{1} & \multirow{4}{*}{$325 \mu \mathrm{m}$} & 30 & \multirow{4}{*}{ Crude oil } & $520(425)$ & $453(338)$ & $419(285)$ & $420(310)$ \\
\hline & & 60 & & $583(439)$ & $490(350)$ & $440(293)$ & $433(290)$ \\
\hline & & 90 & & $575(447)$ & $471(346)$ & $440(295)$ & $410(280)$ \\
\hline & & 120 & & $600(450)$ & $475(348)$ & $438(295)$ & $420(281)$ \\
\hline \multirow{4}{*}{2} & \multirow{4}{*}{$325 \mu \mathrm{m}$} & 30 & \multirow{4}{*}{ Diesel } & $470(336)$ & $433(309)$ & $413(285)$ & $403(273)$ \\
\hline & & 60 & & $480(338)$ & $445(310)$ & $425(286)$ & $413(283)$ \\
\hline & & 90 & & $475(333)$ & $443(310)$ & $423(290)$ & $422(288)$ \\
\hline & & 120 & & $490(345)$ & $449(310)$ & $415(285)$ & $420(286)$ \\
\hline \multirow{4}{*}{3} & \multirow{4}{*}{$325 \mu \mathrm{m}$} & 30 & \multirow{4}{*}{ Kerosene } & $445(322)$ & $425(288)$ & $395(255)$ & $375(245)$ \\
\hline & & 60 & & $453(320)$ & $430(287)$ & $403(261)$ & $398(251)$ \\
\hline & & 90 & & $454(320)$ & $430(288)$ & $403(263)$ & $397(250)$ \\
\hline & & 120 & & $460(314)$ & $434(289)$ & $403(263)$ & $398(253)$ \\
\hline \multirow{4}{*}{4} & \multirow{4}{*}{$325 \mu \mathrm{m}$} & 30 & \multirow{4}{*}{ Petrol } & $375(233)$ & $370(230)$ & $360(200)$ & $330(180)$ \\
\hline & & 60 & & $390(240)$ & $388(232)$ & $368(206)$ & 335 (182) \\
\hline & & 90 & & $392(240)$ & $368(230)$ & 367 (195) & 334 (182) \\
\hline & & 120 & & $399(242)$ & 373 (234) & 367 (198) & 334 (183) \\
\hline \multirow{4}{*}{5} & \multirow{4}{*}{$625 \mu \mathrm{m}$} & 30 & \multirow{4}{*}{ Crude Oil } & $463(338)$ & 445 (293) & $398(255)$ & $388(235)$ \\
\hline & & 60 & & $508(359)$ & $460(298)$ & $408(260)$ & $400(243)$ \\
\hline & & 90 & & $500(357)$ & $463(290)$ & $403(255)$ & $396(241)$ \\
\hline & & 120 & & $530(381)$ & 465 (298) & $410(261)$ & $403(243)$ \\
\hline \multirow{4}{*}{6} & \multirow{4}{*}{$625 \mu \mathrm{m}$} & 30 & \multirow{4}{*}{ Diesel } & $410(296)$ & $404(275)$ & $375(225)$ & 350 (188) \\
\hline & & 60 & & $413(302)$ & 414 (277) & 395 (239) & 365 (191) \\
\hline & & 90 & & $413(294)$ & $400(285)$ & $399(250)$ & 348 (185) \\
\hline & & 120 & & $413(292)$ & $405(285)$ & $398(250)$ & 355 (183) \\
\hline \multirow{4}{*}{7} & \multirow{4}{*}{$625 \mu \mathrm{m}$} & 30 & \multirow{4}{*}{ Kerosene } & $393(258)$ & $390(245)$ & $363(191)$ & $315(163)$ \\
\hline & & 60 & & $405(264)$ & $394(248)$ & 368 (194) & $358(171)$ \\
\hline & & 90 & & $409(262)$ & $393(245)$ & 364 (192) & $353(170)$ \\
\hline & & 120 & & $418(273)$ & $405(248)$ & 378 (199) & $363(178)$ \\
\hline \multirow{4}{*}{8} & \multirow{4}{*}{$625 \mu \mathrm{m}$} & 30 & \multirow{4}{*}{ Petrol } & 373 (188) & $365(180)$ & $335(161)$ & $313(152)$ \\
\hline & & 60 & & $385(208)$ & $370(182)$ & $350(165)$ & $320(154)$ \\
\hline & & 90 & & $383(206)$ & $368(180)$ & 348 (164) & $325(158)$ \\
\hline & & 120 & & $393(213)$ & $373(201)$ & $360(168)$ & $330(168)$ \\
\hline
\end{tabular}

[\% Recovery in parenthesis]. 
Table 2. (a) Percentage absorption/recovery profiles of sorbates by combination of carbonized and uncarbonized goat hair at different particles size and contact time; (b) Calculated percentage absorption/recovery profiles of sorbates by equal combination of carbonized and uncarbonized goat hair at different particles size and contact time.

(a)

\begin{tabular}{|c|c|c|c|c|c|c|c|}
\hline \multicolumn{8}{|c|}{$\%$ Absorption/Recovery } \\
\hline S/No & Particle size & Contact time(min) & Sorbates & $\mathbf{1}^{\text {st }}$ Use & $2^{\text {nd }}$ Use & $3^{\text {rd }}$ Use & $4^{\text {th }}$ Use \\
\hline \multirow{4}{*}{1} & \multirow{4}{*}{$325 \mu \mathrm{m}$} & 30 & \multirow{4}{*}{ Crude oil } & $540(280)$ & $500(220)$ & $480(296)$ & $460(260)$ \\
\hline & & 60 & & $550(280)$ & $540(280)$ & $490(296)$ & $420(228)$ \\
\hline & & 90 & & $620(310)$ & $570(290)$ & $495(305)$ & $450(250)$ \\
\hline & & 120 & & $690(310)$ & $600(300)$ & $495(310)$ & $460(265)$ \\
\hline \multirow{4}{*}{2} & \multirow{4}{*}{$325 \mu \mathrm{m}$} & 30 & \multirow{4}{*}{ Diesel } & $450(260)$ & $430(260)$ & $400(258)$ & $380(242)$ \\
\hline & & 60 & & $450(268)$ & $450(260)$ & $420(260)$ & $395(220)$ \\
\hline & & 90 & & $500(270)$ & $490(268)$ & $430(265)$ & $400(240)$ \\
\hline & & 120 & & $550(380)$ & $530(276)$ & $450(268)$ & $410(250)$ \\
\hline \multirow{4}{*}{3} & \multirow{4}{*}{$325 \mu \mathrm{m}$} & 30 & \multirow{4}{*}{ Kerosene } & $335(190)$ & $330(215)$ & $270(180)$ & $270(160)$ \\
\hline & & 60 & & $375(198)$ & $320(210)$ & $335(190)$ & $290(168)$ \\
\hline & & 90 & & $380(200)$ & $365(220)$ & $340(190)$ & $295(186)$ \\
\hline & & 120 & & $390(205)$ & $380(230)$ & $360(210)$ & $350(210)$ \\
\hline \multirow{4}{*}{4} & \multirow{4}{*}{$325 \mu \mathrm{m}$} & 30 & \multirow{4}{*}{ Petrol } & $275(178)$ & $272(165)$ & $265(172)$ & $265(170)$ \\
\hline & & 60 & & $280(196)$ & $296(200)$ & $275(175)$ & $270(170)$ \\
\hline & & 90 & & $360(198)$ & $355(235)$ & $280(175)$ & $280(172)$ \\
\hline & & 120 & & $380(220)$ & $370(240)$ & $350(190)$ & $320(200)$ \\
\hline \multirow{4}{*}{5} & \multirow{4}{*}{$625 \mu \mathrm{m}$} & 30 & \multirow{4}{*}{ Crude oil } & $426(273)$ & $420(200)$ & $375(200)$ & $375(162)$ \\
\hline & & 60 & & $465(260)$ & $470(210)$ & $390(206)$ & $380(160)$ \\
\hline & & 90 & & $480(266)$ & $475(260)$ & $420(220)$ & $390(175)$ \\
\hline & & 120 & & $650(330)$ & $510(280)$ & $450(225)$ & $395(180)$ \\
\hline \multirow{4}{*}{6} & \multirow{4}{*}{$625 \mu \mathrm{m}$} & 30 & \multirow{4}{*}{ Diesel } & $420(255)$ & $370(180)$ & $350(178)$ & $310(165)$ \\
\hline & & 60 & & $420(250)$ & $400(195)$ & $365(180)$ & $320(170)$ \\
\hline & & 90 & & $430(260)$ & $410(200)$ & $385(190)$ & $370(185)$ \\
\hline & & 120 & & $450(270)$ & $420(210)$ & $390(200)$ & 385 (190) \\
\hline \multirow{4}{*}{7} & \multirow{4}{*}{$625 \mu \mathrm{m}$} & 30 & \multirow{4}{*}{ Kerosene } & $300(170)$ & $285(160)$ & $280(160)$ & $270(150)$ \\
\hline & & 60 & & $360(175)$ & $380(165)$ & $280(162)$ & $275(150)$ \\
\hline & & 90 & & $380(176)$ & $385(165)$ & $286(165)$ & $280(155)$ \\
\hline & & 120 & & $390(180)$ & $390(175)$ & $285(165)$ & $280(154)$ \\
\hline \multirow{4}{*}{8} & \multirow{4}{*}{$625 \mu \mathrm{m}$} & 30 & \multirow{4}{*}{ Petrol } & $260(150)$ & $255(155)$ & $252(150)$ & $248(140)$ \\
\hline & & 60 & & $270(155)$ & $265(160)$ & $260(155)$ & $250(150)$ \\
\hline & & 90 & & $285(160)$ & $280(168)$ & $270(160)$ & $260(148)$ \\
\hline & & 120 & & $300(165)$ & $285(170)$ & $280(165)$ & $265(150)$ \\
\hline
\end{tabular}

[\% Recovery in parenthesis]. 
(b)

\begin{tabular}{|c|c|c|c|c|c|c|c|}
\hline \multicolumn{8}{|c|}{$\%$ Absorption/Recovery } \\
\hline S/No & Particle sizes & Contact time(min) & Sorbates & $\mathbf{1}^{\text {st }}$ Use & $2^{\text {nd }}$ Use & $3^{\text {rd }}$ Use & $4^{\text {th }}$ Use \\
\hline \multirow{4}{*}{1} & \multirow{4}{*}{$325 \mu \mathrm{m}$} & 30 & \multirow{4}{*}{ Crude oil } & $435(314)$ & $431(310)$ & $418(303)$ & $399(258)$ \\
\hline & & 60 & & $453(316)$ & $424(300)$ & $422(308)$ & $405(268)$ \\
\hline & & 90 & & $463(322)$ & $428(306)$ & $425(315)$ & $412(260)$ \\
\hline & & 120 & & $478(324)$ & $435(314)$ & $430(319)$ & $420(278)$ \\
\hline \multirow{4}{*}{2} & \multirow{4}{*}{$325 \mu \mathrm{m}$} & 30 & \multirow{4}{*}{ Diesel } & $408(292)$ & $394(246)$ & $385(203)$ & $363(196)$ \\
\hline & & 60 & & $430(294)$ & $405(250)$ & $393(215)$ & $369(198)$ \\
\hline & & 90 & & $423(284)$ & $394(245)$ & $398(220)$ & $389(204)$ \\
\hline & & 120 & & $434(289)$ & $409(252)$ & $403(231)$ & 395 (209) \\
\hline \multirow{4}{*}{3} & \multirow{4}{*}{$325 \mu \mathrm{m}$} & 30 & \multirow{4}{*}{ Kerosene } & $317(201)$ & $311(199)$ & $305(231)$ & $294(188)$ \\
\hline & & 60 & & $321(202)$ & $313(198)$ & $304(175)$ & $293(188)$ \\
\hline & & 90 & & $327(210)$ & $310(196)$ & $310(178)$ & $296(187)$ \\
\hline & & 120 & & $392(208)$ & $308(201)$ & $314(182)$ & 297 (189) \\
\hline \multirow{4}{*}{4} & \multirow{4}{*}{$325 \mu \mathrm{m}$} & 30 & \multirow{4}{*}{ Petrol } & $306(184)$ & $301(167)$ & $293(168)$ & $280(161)$ \\
\hline & & 60 & & $306(184)$ & 303 (173) & $295(168)$ & $280(162)$ \\
\hline & & 90 & & $315(190)$ & 305 (171) & $298(168)$ & $281(161)$ \\
\hline & & 120 & & 318 (192) & 307 (174) & $297(168)$ & $285(163)$ \\
\hline \multirow{4}{*}{5} & \multirow{4}{*}{$625 \mu \mathrm{m}$} & 30 & \multirow{4}{*}{ Crude Oil } & $319(249)$ & 301 (193) & $293(181)$ & $288(183)$ \\
\hline & & 60 & & $325(253)$ & 324 (209) & $306(186)$ & $296(185)$ \\
\hline & & 90 & & $340(260)$ & 323 (209) & 309 (207) & $292(183)$ \\
\hline & & 120 & & $363(265)$ & 333 (209) & $310(208)$ & 300 (187) \\
\hline \multirow{4}{*}{6} & \multirow{4}{*}{$625 \mu \mathrm{m}$} & 30 & \multirow{4}{*}{ Diesel } & $293(208)$ & $284(180)$ & $282(178)$ & 275 (173) \\
\hline & & 60 & & $296(209)$ & $289(182)$ & $286(231)$ & 284 (179) \\
\hline & & 90 & & 308 (214) & $292(185)$ & $286(181)$ & $280(178)$ \\
\hline & & 120 & & $312(220)$ & 308 (189) & $293(186)$ & $287(182)$ \\
\hline \multirow{4}{*}{7} & \multirow{4}{*}{$625 \mu \mathrm{m}$} & 30 & \multirow{4}{*}{ Kerosene } & $275(181)$ & $271(175)$ & $266(172)$ & 263 (159) \\
\hline & & 60 & & $272(173)$ & $270(175)$ & $269(173)$ & $267(161)$ \\
\hline & & 90 & & 285 (183) & 275 (178) & $271(174)$ & $267(162)$ \\
\hline & & 120 & & $292(185)$ & $288(180)$ & $275(176)$ & $272(165)$ \\
\hline \multirow{4}{*}{8} & \multirow{4}{*}{$625 \mu \mathrm{m}$} & 30 & \multirow{4}{*}{ Petrol } & $244(160)$ & $234(152)$ & $231(143)$ & $228(136)$ \\
\hline & & 60 & & $240(158)$ & $236(154)$ & $232(143)$ & $230(138)$ \\
\hline & & 90 & & $243(158)$ & $240(155)$ & $233(144)$ & 229 (137) \\
\hline & & 120 & & $250(162)$ & $242(156)$ & $234(143)$ & 231 (139) \\
\hline
\end{tabular}

[\% Recovery in parenthesis]. 
of sorbates by equal combination of carbonized and uncarbonized goat hair at different particles size and contact time. Considering absorption/recovery when using $325 \mu \mathrm{m}$, crude oil was absorbed and recovered highest at 120 mins contact time for all application.

Diesel followed the same trend. Kerosene was absorbed highest at 120 mins for all tests but was recovered most at $2^{\text {nd }}$ use from 30 to 120 mins contact time. The same was seen in petrol. Using $625 \mu \mathrm{m}$, there was a near consistency in highest absorption and recovery at 120 mins contact time at all applications; the same could be said of diesel. Baring minor variation the same was observed for kerosene and diesel.

Table 2(b): Calculated percentage absorption/recovery profiles of sorbates by equal combination of carbonized and uncarbonized goat hair at different particles size and contact time. Considering $325 \mu \mathrm{m}$, crude oil was absorbed/recovered highest at 120 mins contact time for all the four (4) applications, that of diesel followed the same trend. Kerosene was sorbed most at 120 mins and 60 mins for $1^{\text {st }}$ and $2^{\text {nd }}$ application while recovery was more at 90 mins at $1^{\text {st }}$ use. Petrol was nearly equally absorbed and recovered all through at 120 mins, except for minor variations. Using $625 \mu \mathrm{m}$, crude oil, diesel, kerosene and petrol were sorbed/recovered more at $120 \mathrm{mins}$ contact time for all applications, except for insignificant differences.

Table 3(a): Percentage absorption/recovery profiles of sorbates by equal combination of carbonized and uncarbonized coco-nut husk at different particles size and contact time, when considering $325 \mu \mathrm{m}$, a trend showing that the longer the contact time (120 mins) the higher the absorption/recovery for the sorbates by the combined sorbent at any contact time and use except for kerosene where highest recovery at $2^{\text {nd }}$ use occurred at 30 mins. Using $625 \mu \mathrm{m}$, crude oil was absorbed/recovered highest at 120 mins time, the same with diesel except that highest adsorption for diesel during $3^{\text {rd }}$ use occurred at 90 mins. Kerosene and petrol were absorbed and recovered highest at 120 mins for all applications.

Table 3(b): Calculated percentage absorption/recovery profiles of sorbates by equal combination of carbonized and uncarbonized coco-nut husk at different particles size and contact time, with $325 \mu \mathrm{m}$ highest absorption/recovery for crude oil, diesel and kerosene were at 120 mins except that diesel was recovered most during $3^{\text {rd }}$ use at 90 mins, petrol was absorbed more when $1^{\text {st }}$ used at 90 mins and recovered most at 120 mins. If $625 \mu \mathrm{m}$ is considered, except for minor acceptable differences, crude oil, kerosene and petrol, followed the usual trend of highest absorption/recovery at 120 mins contact time.

Table 4(a): Percentage absorption/ recovery profiles of sorbates by equal combination of carbonized and uncarbonized corn-cob at different particle size and contact time. With $325 \mu \mathrm{m}$, crude oil was absorbed/recovered highest at 120 mins except for $3^{\text {rd }}$ use where highest absorption/recovery occurred at 60 mins. Diesel was absorbed highest at 120 mins in all experiment but recovered more at $60 \mathrm{~min}$ at $1^{\text {st }}$ use. Kerosene and petrol followed already established trend of highest absorption/recovery at longest contact time. Using $625 \mu \mathrm{m}$, crude oil, diesel, kerosene and petrol were absorbed/recovered highest at 120 mins contact time for all applications.

Table 4(b): Calculated percentage absorption/recovery profiles of sorbates by equal combination of carbonized and uncarbonized corn-cob at different particles size and contact time, considering the sorbent of $325 \mu \mathrm{m}$, crude oil was sorbed most at 120 mins for the $1^{\text {st }}$ and $2^{\text {nd }}$ use, recovery for both were at $30 \mathrm{mins}$. Diesel were sorbed at 60 mins for $1^{\text {st }}$ and $2^{\text {nd }}$ use but recovered highest at 30 min, kerosene was absorbed at $30 \mathrm{~min}$ and $60 \mathrm{mins}\left(1^{\text {st }}\right.$ and $2^{\text {nd }}$ use), while highest recovery for both were at 60 and 90 mins. Petrol was sorbed most at 90 mins and 60 mins during earliest use, the same for recovery.

Using $625 \mu \mathrm{m}$, crude oil was sorbed/ recovered highest at 30 mins and 120 mins for $1^{\text {st }}$ and $2^{\text {nd }}$ use, diesel was sorbed and recovered highest at 120 and $30 \mathrm{mins}$ for $1^{\text {st }}$ and $2^{\text {nd }}$ use, that of kerosene was at 60 and 30 mins for both $1^{\text {st }}$ and $2^{\text {nd }}$ use. Petrol was absorbed highest at 30 and 90 mins while recovery was highest at 60,90 and 120 respectively for $2^{\text {nd }}$ and $3^{\text {rd }}$ use.

Table 5: \% retention profiles of sorbates by combined carbonized and uncarbonized feather from experimental and calculated values at different particles size and contact time. $325 \mu \mathrm{m}$, highest experimental retention of crude oil occurred at 120 mins for all use, that of calculated value occurred at 120 mins except for $2^{\text {nd }}$ use where it occurred at 60 mins. Experimental and calculated retention of diesel was highest at 120 mins for $1^{\text {st }}$ and $2^{\text {nd }}$ use while the highest for $3^{\text {rd }}$ and $4^{\text {th }}$ use occurred at 60 and 120 mins respectively. Kerosene was retained highest considering experimental values at 90 mins at $1^{\text {st }}$ use, 60 mins at $2^{\text {nd }}$ use, calculated retained highest at 120 mins at $1^{\text {st }}$ and $2^{\text {nd }}$ use. Petrol was retained at $90 \mathrm{~min}$ during $2^{\text {nd }}$ and $3^{\text {rd }}$ use, variation exists for calculated value. Using $625 \mu \mathrm{m}$, highest retention was at $120\left(1^{\text {st }}\right.$ and $4^{\text {th }}$ ) use and at 30 and 60 mins for $2^{\text {nd }}$ and $3^{\text {rd }}$ use. Calculated retention value has most of the highest at 120 mins, diesel was retained at 60,90 and 120 mins, during $1^{\text {st }}, 2^{\text {nd }}$ and $4^{\text {th }}$ use considering experimental value, calculated retention values varied for each of the contact time. Kerosene was highest at 60 and 90 mins $\left(1^{\text {st }}\right.$ and $\left.2^{\text {nd }}\right)$ use for experiment while calculated value has highest retention at 120 mins at least from $1^{\text {st }}$ to $3^{\text {rd }}$ use. Petrol was retained highest from 90 mins upwards at all applications given experimental value that of calculated varied.

Table 6: percentage retention profiles of sorbates by 
Table 3. (a) Percentage absorption/recovery profiles of sorbates by equal combination of carbonized and uncarbonized coconut husk at different particles size and contact time; (b) Calculated percentage absorption/recovery profiles of sorbates by equal combination of carbonized and uncarbonized coconut husk at different particles size and contact time.

(a)

\begin{tabular}{|c|c|c|c|c|c|c|c|}
\hline \multicolumn{8}{|c|}{$\%$ Absorption/Recovery } \\
\hline S/No & Particles Size & Contact Time & Sorbate & $\mathbf{1}^{\text {st }}$ Use & $2^{\text {nd }}$ Use & $3^{\text {rd }}$ Use & $4^{\text {th }}$ Use \\
\hline \multirow{4}{*}{1} & \multirow{4}{*}{$325 \mu \mathrm{m}$} & 30 & \multirow{4}{*}{ Crude oil } & $510(250)$ & $480(280)$ & $460(258)$ & $450(250)$ \\
\hline & & 60 & & $560(280)$ & $500(300)$ & $470(280)$ & $455(250)$ \\
\hline & & 90 & & $610(300)$ & $550(290)$ & $480(280)$ & $470(260)$ \\
\hline & & 120 & & $630(305)$ & $560(310)$ & $485(290)$ & $480(270)$ \\
\hline \multirow{4}{*}{2} & \multirow{4}{*}{$325 \mu \mathrm{m}$} & 30 & \multirow{4}{*}{ Diesel } & $385(205)$ & $380(180)$ & $380(180)$ & $370(180)$ \\
\hline & & 60 & & $420(220)$ & $400(190)$ & $390(170)$ & $380(190)$ \\
\hline & & 90 & & $465(215)$ & $410(210)$ & $400(190)$ & $395(195)$ \\
\hline & & 120 & & $500(260)$ & $430(215)$ & $400(200)$ & $405(200)$ \\
\hline \multirow{4}{*}{3} & \multirow{4}{*}{$325 \mu \mathrm{m}$} & 30 & \multirow{4}{*}{ Kerosene } & $320(180)$ & $310(200)$ & $260(150)$ & $260(154)$ \\
\hline & & 60 & & $360(190)$ & $310(185)$ & $285(170)$ & $270(160)$ \\
\hline & & 90 & & $365(200)$ & $320(180)$ & $290(180)$ & $280(170)$ \\
\hline & & 120 & & $380(205)$ & $330(186)$ & $330(190)$ & $290(185)$ \\
\hline \multirow{4}{*}{4} & \multirow{4}{*}{$325 \mu \mathrm{m}$} & 30 & \multirow{4}{*}{ Petrol } & $260(150)$ & $260(150)$ & $254(150)$ & $240(150)$ \\
\hline & & 60 & & $280(160)$ & $285(175)$ & $265(170)$ & $260(155)$ \\
\hline & & 90 & & $295(162)$ & $290(180)$ & $270(170)$ & $275(160)$ \\
\hline & & 120 & & $350(195)$ & $340(190)$ & $320(198)$ & $300(185)$ \\
\hline \multirow{4}{*}{5} & \multirow{4}{*}{$625 \mu \mathrm{m}$} & 30 & \multirow{4}{*}{ Crude Oil } & $460(270)$ & $410(285)$ & $370(170)$ & 368 (190) \\
\hline & & 60 & & $500(285)$ & $450(290)$ & $385(175)$ & 375 (192) \\
\hline & & 90 & & $485(280)$ & $470(285)$ & $390(180)$ & 390 (195) \\
\hline & & 120 & & $630(310)$ & $500(315)$ & $400(260)$ & $390(200)$ \\
\hline \multirow{4}{*}{6} & \multirow{4}{*}{$625 \mu \mathrm{m}$} & 30 & \multirow{4}{*}{ Diesel } & $380(180)$ & $350(180)$ & $320(165)$ & $305(162)$ \\
\hline & & 60 & & $396(185)$ & $360(190)$ & $330(170)$ & $310(165)$ \\
\hline & & 90 & & $400(255)$ & $380(195)$ & 395 (185) & $360(175)$ \\
\hline & & 120 & & $430(265)$ & $410(260)$ & $390(180)$ & $375(180)$ \\
\hline \multirow{4}{*}{7} & \multirow{4}{*}{$625 \mu \mathrm{m}$} & 30 & \multirow{4}{*}{ Kerosene } & $285(156)$ & $280(162)$ & $280(160)$ & $270(158)$ \\
\hline & & 60 & & $290(160)$ & $285(165)$ & $275(156)$ & $268(154)$ \\
\hline & & 90 & & $290(162)$ & $290(170)$ & $278(158)$ & $275(150)$ \\
\hline & & 120 & & $310(185))$ & $300(170)$ & $285(160)$ & $275(160)$ \\
\hline \multirow{4}{*}{8} & \multirow{4}{*}{$625 \mu \mathrm{m}$} & 30 & \multirow{4}{*}{ Petrol } & $250(146)$ & $250(150)$ & $248(145)$ & $246(145)$ \\
\hline & & 60 & & $270(150)$ & $260(150)$ & $260(150)$ & $250(150)$ \\
\hline & & 90 & & $285(165)$ & $275(160)$ & $275(155)$ & $260(150)$ \\
\hline & & 120 & & $290(175)$ & $285(165)$ & $280(165)$ & $260(155)$ \\
\hline
\end{tabular}

[\% Recovery in parenthesis]. 
(b)

\begin{tabular}{|c|c|c|c|c|c|c|c|}
\hline \multicolumn{8}{|c|}{$\%$ Absorption/Recovery } \\
\hline S/No & Particle size & Contact time (min) & Sorbates & $1^{\text {st }}$ Use & $2^{\text {nd }}$ Use & $3^{\text {rd }}$ Use & $4^{\text {th }}$ Use \\
\hline \multirow{4}{*}{1} & \multirow{4}{*}{$325 \mu \mathrm{m}$} & 30 & \multirow{4}{*}{ Crude oil } & 405 (299) & $386(213)$ & $357(196)$ & $293(180)$ \\
\hline & & 60 & & $427(300)$ & $395(217)$ & $359(191)$ & $285(174)$ \\
\hline & & 90 & & $426(304)$ & $403(244)$ & $359(193)$ & $292(178)$ \\
\hline & & 120 & & $435(307)$ & $418(260)$ & $366(205)$ & $304(183)$ \\
\hline \multirow{4}{*}{2} & \multirow{4}{*}{$325 \mu \mathrm{m}$} & 30 & \multirow{4}{*}{ Diesel } & $394(240)$ & $328(210)$ & $300(169)$ & $278(149)$ \\
\hline & & 60 & & $394(224)$ & $330(206)$ & $305(172)$ & $280(150)$ \\
\hline & & 90 & & $399(220)$ & $334(202)$ & $308(173)$ & $278(152)$ \\
\hline & & 120 & & $410(228)$ & $338(210)$ & $308(167)$ & $288(158)$ \\
\hline \multirow{4}{*}{3} & \multirow{4}{*}{$325 \mu \mathrm{m}$} & 30 & \multirow{4}{*}{ Kerosene } & $285(171)$ & $274(158)$ & $272(156)$ & $265(154)$ \\
\hline & & 60 & & $285(168)$ & $282(163)$ & $276(157)$ & $268(156)$ \\
\hline & & 90 & & $278(172)$ & $278(160)$ & $278(160)$ & $274(158)$ \\
\hline & & 120 & & $292(179)$ & $282(163)$ & $278(161)$ & $279(159)$ \\
\hline \multirow{4}{*}{4} & \multirow{4}{*}{$325 \mu \mathrm{m}$} & 30 & \multirow{4}{*}{ Petrol } & 275 (169) & $266(160)$ & $258(148)$ & 255 (140) \\
\hline & & 60 & & 277 (167) & $269(162)$ & $264(151)$ & $259(141)$ \\
\hline & & 90 & & $280(176)$ & $269(162)$ & $265(154)$ & $258(144)$ \\
\hline & & 120 & & $278(178)$ & $272(162)$ & $267(155)$ & $260(146)$ \\
\hline \multirow{4}{*}{5} & \multirow{4}{*}{$625 \mu \mathrm{m}$} & 30 & \multirow{4}{*}{$\begin{array}{c}\text { Crude } \\
\text { Oil }\end{array}$} & $323(184)$ & $305(170)$ & $286(174)$ & 264 (164) \\
\hline & & 60 & & $332(187)$ & $311(173)$ & $288(176)$ & $272(166)$ \\
\hline & & 90 & & 337 (191) & $313(172)$ & $291(176)$ & $270(168)$ \\
\hline & & 120 & & 340 (198) & 315 (177) & 294 (179) & 277 (169) \\
\hline \multirow{4}{*}{6} & \multirow{4}{*}{$625 \mu \mathrm{m}$} & 30 & \multirow{4}{*}{ Diesel } & $280(165)$ & 267 (164) & $261(159)$ & 258 (144) \\
\hline & & 60 & & $283(167)$ & $267(163)$ & $264(160)$ & $260(147)$ \\
\hline & & 90 & & $281(165)$ & $267(163)$ & $264(161)$ & 264 (147) \\
\hline & & 120 & & $287(168)$ & $272(167)$ & $268(163)$ & $265(148)$ \\
\hline \multirow{4}{*}{7} & \multirow{4}{*}{$625 \mu \mathrm{m}$} & 30 & \multirow{4}{*}{ Kerosene } & $264(166)$ & $250(148)$ & $218(142)$ & 204 (111) \\
\hline & & 60 & & $269(170)$ & $255(148)$ & $219(143)$ & $206(111)$ \\
\hline & & 90 & & $278(173)$ & $258(149)$ & $222(144)$ & $206(114)$ \\
\hline & & 120 & & $282(175)$ & $259(153)$ & $223(145)$ & 211 (117) \\
\hline \multirow{4}{*}{8} & \multirow{4}{*}{$625 \mu \mathrm{m}$} & 30 & \multirow{4}{*}{ Petrol } & $211(125)$ & 198 (117) & $192(108)$ & $183(101)$ \\
\hline & & 60 & & $214(126)$ & 199 (118) & $196(109)$ & $185(102)$ \\
\hline & & 90 & & $214(126)$ & 199 (117) & $196(108)$ & $188(105)$ \\
\hline & & 120 & & $220(130)$ & $201(118)$ & $195(108)$ & $189(105)$ \\
\hline
\end{tabular}

[\% Recovery in parenthesis]. 
Table 4. (a) Percentage absorption/recovery profiles of sorbates by equal combination of carbonized and uncarbonized corn-cob at different particles size and contact time; (b) Calculated percentage absorption/recovery profiles of sorbates by equal combination of carbonized and uncarbonized corn-cob at different particles size and contact time.

(a)

\begin{tabular}{|c|c|c|c|c|c|c|c|}
\hline \multicolumn{8}{|c|}{$\%$ Absorption/Recovery } \\
\hline S/No & Particle Size & Contact time (min) & Sorbates & $\mathbf{1}^{\text {st }}$ Use & $2^{\text {nd }}$ Use & $3^{\text {rd }}$ Use & $4^{\text {th }}$ Use \\
\hline \multirow{4}{*}{1} & \multirow{4}{*}{$325 \mu \mathrm{m}$} & 30 & \multirow{4}{*}{ Crude oil } & $490(298)$ & $460(292)$ & $430(250)$ & $450(280)$ \\
\hline & & 60 & & $500(380)$ & $480(300)$ & $440(250)$ & $460(288)$ \\
\hline & & 90 & & $520(290)$ & $490(298)$ & $370(220)$ & $440(260)$ \\
\hline & & 120 & & $600(315)$ & $550(392)$ & $360(220)$ & $470(296)$ \\
\hline \multirow{4}{*}{2} & \multirow{4}{*}{$325 \mu \mathrm{m}$} & 30 & \multirow{4}{*}{ Diesel } & $386(220)$ & $273(138)$ & $313(188)$ & $366(198)$ \\
\hline & & 60 & & $406(246)$ & $373(220)$ & $380(220)$ & $393(213)$ \\
\hline & & 90 & & $387(233)$ & $413(226)$ & $395(223)$ & $395(213)$ \\
\hline & & 120 & & $480(206)$ & $413(235)$ & $400(240)$ & $400(233)$ \\
\hline \multirow{4}{*}{3} & \multirow{4}{*}{$325 \mu \mathrm{m}$} & 30 & \multirow{4}{*}{ Kerosene } & $292(188)$ & $294(192)$ & $252(168)$ & $272(168)$ \\
\hline & & 60 & & $296(190)$ & $296(198)$ & $260(172)$ & $292(168)$ \\
\hline & & 90 & & $298(204)$ & 294 (198) & $276(186)$ & $294(184)$ \\
\hline & & 120 & & $340(220)$ & $336(208)$ & $332(204)$ & $344(204)$ \\
\hline \multirow{4}{*}{4} & \multirow{4}{*}{$325 \mu \mathrm{m}$} & 30 & \multirow{4}{*}{ Petrol } & $260(1540$ & $290(205)$ & $240(143)$ & $270(170)$ \\
\hline & & 60 & & $275(185)$ & $295(210)$ & $260(170)$ & $290(180)$ \\
\hline & & 90 & & 285 (195) & $305(230)$ & $275(180)$ & $290(185)$ \\
\hline & & 120 & & $340(215)$ & $330(280)$ & $310(210)$ & $340(215)$ \\
\hline \multirow{4}{*}{5} & \multirow{4}{*}{$625 \mu \mathrm{m}$} & 30 & \multirow{4}{*}{ Crude Oil } & $420(260)$ & $400(289)$ & $380(160)$ & $370(160)$ \\
\hline & & 60 & & $480(280)$ & $460(290)$ & $375(162)$ & $380(160)$ \\
\hline & & 90 & & $460(265)$ & $470(298)$ & $385(165)$ & $378(158)$ \\
\hline & & 120 & & $610(330)$ & $500(398)$ & $390(170)$ & 385 (165) \\
\hline \multirow{4}{*}{6} & \multirow{4}{*}{$625 \mu \mathrm{m}$} & 30 & \multirow{4}{*}{ Diesel } & $330(178)$ & $326(170)$ & $300(170)$ & $300(160)$ \\
\hline & & 60 & & $340(180)$ & 348 (186) & 315 (176) & 305 (166) \\
\hline & & 90 & & $390(188)$ & $360(190)$ & $380(188)$ & $366(170)$ \\
\hline & & 120 & & $420(260)$ & $406(260)$ & $388(190)$ & $370(180)$ \\
\hline \multirow{4}{*}{7} & \multirow{4}{*}{$625 \mu \mathrm{m}$} & 30 & \multirow{4}{*}{ Kerosene } & $280(158)$ & $280(160)$ & $278(158)$ & $260(154)$ \\
\hline & & 60 & & $285(160)$ & $282(166)$ & $276(160)$ & $268(158)$ \\
\hline & & 90 & & $290(164)$ & $285(170)$ & $280(160)$ & $270(160)$ \\
\hline & & 120 & & $300(180)$ & $290(172)$ & $288(166)$ & $280(170)$ \\
\hline \multirow{4}{*}{8} & \multirow{4}{*}{$625 \mu \mathrm{m}$} & 30 & \multirow{4}{*}{ Petrol } & $250(148)$ & $252(150)$ & $250(148)$ & $240(146)$ \\
\hline & & 60 & & $270(156)$ & $260(154)$ & $258(154)$ & $250(150)$ \\
\hline & & 90 & & $280(166)$ & $270(260)$ & $266(162)$ & $260(152)$ \\
\hline & & 120 & & $290(170)$ & $288(165)$ & $280(168)$ & $266(154)$ \\
\hline
\end{tabular}

[\% Recovery in parenthesis]. 
(b)

\begin{tabular}{|c|c|c|c|c|c|c|c|}
\hline \multicolumn{8}{|c|}{$\%$ Absorption/Recovery } \\
\hline S/No & Particle size & Contact time (min) & Sorbates & $1^{\text {st }}$ Use & $2^{\text {nd }}$ Use & $3^{\text {rd }}$ Use & $4^{\text {th }}$ Use \\
\hline \multirow{4}{*}{1} & \multirow{4}{*}{$325 \mu \mathrm{m}$} & 30 & \multirow{4}{*}{ Crude oil } & $391(286)$ & $375(243)$ & $354(210)$ & $287(186)$ \\
\hline & & 60 & & $389(281)$ & $378(222)$ & $356(194)$ & $297(186)$ \\
\hline & & 90 & & $388(238)$ & $361(210)$ & $353(207)$ & $323(194)$ \\
\hline & & 120 & & $402(272)$ & $381(239)$ & $353(208)$ & 293 (189) \\
\hline \multirow{4}{*}{2} & \multirow{4}{*}{$325 \mu \mathrm{m}$} & 30 & \multirow{4}{*}{ Diesel } & $331(246)$ & $321(243)$ & $264(172)$ & $226(130)$ \\
\hline & & 60 & & $380(238)$ & $368(220)$ & $268(159)$ & $210(125)$ \\
\hline & & 90 & & $316(209)$ & $340(198)$ & $268(159)$ & $209(131)$ \\
\hline & & 120 & & $318(223)$ & $323(193)$ & $293(190)$ & $210(133)$ \\
\hline \multirow{4}{*}{3} & \multirow{4}{*}{$325 \mu \mathrm{m}$} & 30 & \multirow{4}{*}{ Kerosene } & $280(171)$ & $266(143)$ & $270(106)$ & $260(106)$ \\
\hline & & 60 & & $310(195)$ & $268(134)$ & $275(108)$ & $275(111)$ \\
\hline & & 90 & & $283(157)$ & $283(150)$ & $260(133)$ & $253(101)$ \\
\hline & & 120 & & $300(161)$ & $240(111)$ & $258(111)$ & $248(107)$ \\
\hline \multirow{4}{*}{4} & \multirow{4}{*}{$325 \mu \mathrm{m}$} & 30 & \multirow{4}{*}{ Petrol } & $228(125)$ & $241(117)$ & $205(112)$ & $160(85)$ \\
\hline & & 60 & & $250(148)$ & $250(118)$ & $218(113)$ & $168(91)$ \\
\hline & & 90 & & $269(170)$ & $240(118)$ & $215(110)$ & $137(81)$ \\
\hline & & 120 & & $265(168)$ & $233(117)$ & $216(111)$ & $148(77)$ \\
\hline \multirow{4}{*}{5} & \multirow{4}{*}{$625 \mu \mathrm{m}$} & 30 & \multirow{4}{*}{ Crude Oil } & $315(205)$ & $273(184)$ & $228(145)$ & $203(140)$ \\
\hline & & 60 & & $293(198)$ & 285 (199) & $243(156)$ & 205 (130) \\
\hline & & 90 & & $310(202)$ & $280(181)$ & $237(151)$ & 207 (134) \\
\hline & & 120 & & 306 (199) & 288 (209) & $243(155)$ & 216 (139) \\
\hline \multirow{4}{*}{6} & \multirow{4}{*}{$625 \mu \mathrm{m}$} & 30 & \multirow{4}{*}{ Diesel } & $263(165)$ & $250(135)$ & $163(103)$ & $148(89)$ \\
\hline & & 60 & & $263(163)$ & $235(118)$ & $165(105)$ & $143(88)$ \\
\hline & & 90 & & $270(171)$ & $215(112)$ & $163(105)$ & $148(91)$ \\
\hline & & 120 & & $278(174)$ & $220(118)$ & $163(102)$ & $140(88)$ \\
\hline \multirow{4}{*}{7} & \multirow{4}{*}{$625 \mu \mathrm{m}$} & 30 & \multirow{4}{*}{ Kerosene } & $240(153)$ & $225(128)$ & $189(101)$ & $173(88)$ \\
\hline & & 60 & & $245(143)$ & $225(126)$ & $173(100)$ & $174(89)$ \\
\hline & & 90 & & $238(139)$ & $214(120)$ & $173(960$ & $165(83)$ \\
\hline & & 120 & & $240(136)$ & $223(120)$ & $175(95)$ & $169(84)$ \\
\hline \multirow{4}{*}{8} & \multirow{4}{*}{$625 \mu \mathrm{m}$} & 30 & \multirow{4}{*}{ Petrol } & $205(101)$ & $172(101)$ & $115(97)$ & $107(84)$ \\
\hline & & 60 & & 207 (110) & $170(104)$ & $130(95)$ & $108(89)$ \\
\hline & & 90 & & $213(104)$ & $165(99)$ & $124(92)$ & $107(87)$ \\
\hline & & 120 & & $203(106)$ & $168(104)$ & $131(95)$ & $109(88)$ \\
\hline
\end{tabular}

[\% Recovery in parenthesis]. 
Table 5. Percentage retention profiles of sorbates by combined carbonized and uncarbonized feather from experimental and calculated values at different particles size and contact time.

\begin{tabular}{|c|c|c|c|c|c|c|c|}
\hline \multicolumn{8}{|c|}{$\%$ Retention } \\
\hline S/No & Particle size & Contact time & Sorbates & $\mathbf{1}^{\text {st }}$ Use & $2^{\text {nd }}$ Use & $3^{\text {rd }}$ Use & $4^{\text {th }}$ Use \\
\hline \multirow{4}{*}{1} & \multirow{4}{*}{$325 \mu \mathrm{m}$} & 30 & \multirow{4}{*}{ Crude Oil } & $180(95)$ & $170(115)$ & $135(134)$ & $130(110)$ \\
\hline & & 60 & & $280(144)$ & $260(140)$ & $210(147)$ & $105(143)$ \\
\hline & & 90 & & $300(128)$ & $300(125)$ & $255(145)$ & $95(130)$ \\
\hline & & 120 & & $310(150)$ & $310(127)$ & $300(143)$ & 135 (139) \\
\hline \multirow{4}{*}{2} & \multirow{4}{*}{$325 \mu \mathrm{m}$} & 30 & \multirow{4}{*}{ Diesel } & $150(134)$ & $230(124)$ & $220(128)$ & $135(130)$ \\
\hline & & 60 & & $175(142)$ & $235(135)$ & $230(139)$ & $130(130)$ \\
\hline & & 90 & & $190(142)$ & $230(133)$ & $205(133)$ & $145(134)$ \\
\hline & & 120 & & $200(145)$ & $255(139)$ & $210(130)$ & $160(134)$ \\
\hline \multirow{4}{*}{3} & \multirow{4}{*}{$325 \mu \mathrm{m}$} & 30 & \multirow{4}{*}{ Kerosene } & $165(123)$ & $150(137)$ & $120(140)$ & $180(130)$ \\
\hline & & 60 & & $180(133)$ & $180(143)$ & $140(142)$ & $150(147)$ \\
\hline & & 90 & & $190(134)$ & $170(142)$ & $175(140)$ & $160(147)$ \\
\hline & & 120 & & $145(146)$ & $170(145)$ & $150(140)$ & $170(145)$ \\
\hline \multirow{4}{*}{4} & \multirow{4}{*}{$325 \mu \mathrm{m}$} & 30 & \multirow{4}{*}{ Petrol } & $130(142)$ & $180(140)$ & $195(160)$ & $200(150)$ \\
\hline & & 60 & & $150(150)$ & $186(156)$ & $190(162)$ & $197(153)$ \\
\hline & & 90 & & $145(152)$ & $200(138)$ & $210(172)$ & $205(152)$ \\
\hline & & 120 & & $150(157)$ & 190 (139) & $202(169)$ & $210(151)$ \\
\hline \multirow{4}{*}{5} & \multirow{4}{*}{$625 \mu \mathrm{m}$} & 30 & \multirow{4}{*}{ Crude Oil } & $145(125)$ & $130(152)$ & $215(143)$ & $195(153)$ \\
\hline & & 60 & & $165(149)$ & $116(162)$ & $240 \quad(148)$ & $195(157)$ \\
\hline & & 90 & & $195(143)$ & $125(173)$ & $225(148)$ & $210(155)$ \\
\hline & & 120 & & $280(149)$ & $120(167)$ & 165 (149) & $225(160)$ \\
\hline \multirow{4}{*}{6} & \multirow{4}{*}{$625 \mu \mathrm{m}$} & 30 & \multirow{4}{*}{ Diesel } & $130(114)$ & 130 (129) & $190(1500$ & $160(162)$ \\
\hline & & 60 & & $165(111)$ & 140 (137) & $200(156)$ & $180(174)$ \\
\hline & & 90 & & $145(119)$ & $155(115)$ & $205(149)$ & $210(163)$ \\
\hline & & 120 & & $164(121)$ & $145(120)$ & $215(148)$ & $206(172)$ \\
\hline \multirow{4}{*}{7} & \multirow{4}{*}{$625 \mu \mathrm{m}$} & 30 & \multirow{4}{*}{ Kerosene } & $180(135)$ & $190(145)$ & $190(172)$ & $190(152)$ \\
\hline & & 60 & & $215(139)$ & $200(146)$ & $210(174)$ & 205 (187) \\
\hline & & 90 & & $175(147)$ & $225(148)$ & $210(172)$ & $205(183)$ \\
\hline & & 120 & & $170(145)$ & $210(157)$ & 205 (179) & $200(185)$ \\
\hline \multirow{4}{*}{8} & \multirow{4}{*}{$625 \mu \mathrm{m}$} & 30 & \multirow{4}{*}{ Petrol } & $185(185)$ & $185(185)$ & $190(174)$ & $150(161)$ \\
\hline & & 60 & & $180(177)$ & $192(188)$ & $190(185)$ & $172(166)$ \\
\hline & & 90 & & $205(177)$ & $200(188)$ & $200(184)$ & $178(167)$ \\
\hline & & 120 & & $205(180)$ & $195(172)$ & 205 (192) & $190(162)$ \\
\hline
\end{tabular}

[\% Retention of sorbates from calculated values in parenthesis]. 
Table 6. Percentage retention profiles of sorbates by combined carbonized and uncarbonized goat hair from experimental and calculated values at different particles size and contact time.

\begin{tabular}{|c|c|c|c|c|c|c|c|}
\hline \multicolumn{8}{|c|}{$\%$ Retention } \\
\hline S/No & Particle size & Contact time & Sorbates & $\mathbf{1}^{\text {st }}$ Use & $2^{\text {nd }}$ Use & $3^{\text {rd }}$ Use & $4^{\text {th }}$ Use \\
\hline \multirow{4}{*}{1} & \multirow{4}{*}{$325 \mu \mathrm{m}$} & 30 & \multirow{4}{*}{ Crude Oil } & $260(121)$ & $280(121)$ & $186(115)$ & $200(141)$ \\
\hline & & 60 & & $270(137)$ & $260(124)$ & $196(114)$ & $192(137)$ \\
\hline & & 90 & & $310(141)$ & $290(122)$ & $190(110)$ & $200(152)$ \\
\hline & & 120 & & $380(154)$ & $300(121)$ & $185(111)$ & $195(142)$ \\
\hline \multirow{4}{*}{2} & \multirow{4}{*}{$325 \mu \mathrm{m}$} & 30 & \multirow{4}{*}{ Diesel } & $190(116)$ & $170(148)$ & $142(182)$ & $138(167)$ \\
\hline & & 60 & & $182(136)$ & $190(155)$ & $160(178)$ & 175 (171) \\
\hline & & 90 & & 230 (139) & $222(149)$ & $165(178)$ & $160(185)$ \\
\hline & & 120 & & $170(145)$ & $254(157)$ & $182(172)$ & $160(186)$ \\
\hline \multirow{4}{*}{3} & \multirow{4}{*}{$325 \mu \mathrm{m}$} & 30 & \multirow{4}{*}{ Kerosene } & $145(116)$ & $115(112)$ & $90(74)$ & $110(106)$ \\
\hline & & 60 & & 177 (119) & $110(115)$ & $145(129)$ & $122(105)$ \\
\hline & & 90 & & $180(117)$ & $145(114)$ & $150(132)$ & 109 (109) \\
\hline & & 120 & & $185(184)$ & $150(107)$ & $150(132)$ & $140(108)$ \\
\hline \multirow{4}{*}{4} & \multirow{4}{*}{$325 \mu \mathrm{m}$} & 30 & \multirow{4}{*}{ Petrol } & 97 (122) & $107(134)$ & $93(125)$ & 95 (119) \\
\hline & & 60 & & $84(122)$ & $96(130)$ & $100(127)$ & $100(118)$ \\
\hline & & 90 & & $162(125)$ & $120(134)$ & $105(130)$ & $108(120)$ \\
\hline & & 120 & & $160(126)$ & $130(124)$ & $160(129)$ & $120(122)$ \\
\hline \multirow{4}{*}{5} & \multirow{4}{*}{$625 \mu \mathrm{m}$} & 30 & \multirow{4}{*}{ Crude Oil } & $153(70)$ & $220(108)$ & $175(112)$ & $213(105)$ \\
\hline & & 60 & & $205(72)$ & $260(115)$ & $184(120)$ & $220(111)$ \\
\hline & & 90 & & $214(80)$ & $260(114)$ & $200(102)$ & 215 (109) \\
\hline & & 120 & & $320(98)$ & $230(124)$ & $225(102)$ & 215 (113) \\
\hline \multirow{4}{*}{6} & \multirow{4}{*}{$625 \mu \mathrm{m}$} & 30 & \multirow{4}{*}{ Diesel } & $165(85)$ & $190(104)$ & $172(104)$ & 145 (102) \\
\hline & & 60 & & $170(87)$ & 205 (107) & $185(55)$ & $150(105)$ \\
\hline & & 90 & & $170(94)$ & $210(107)$ & $195(105)$ & 185 (102) \\
\hline & & 120 & & $180(92)$ & 210) (119) & $190(107)$ & 195 (105) \\
\hline \multirow{4}{*}{7} & \multirow{4}{*}{$625 \mu \mathrm{m}$} & 30 & \multirow{4}{*}{ Kerosene } & $130(94)$ & $125(96)$ & $120(94)$ & $120(104)$ \\
\hline & & 60 & & 185 (99) & $215(95)$ & $118(96)$ & $125(106)$ \\
\hline & & 90 & & 204 (102) & $220(97)$ & $121(97)$ & $125(105)$ \\
\hline & & 120 & & $210(107)$ & $215(108)$ & $120(99)$ & $126(107)$ \\
\hline \multirow{4}{*}{8} & \multirow{4}{*}{$625 \mu \mathrm{m}$} & 30 & \multirow{4}{*}{ Petrol } & $110(84)$ & $100(82)$ & $102(88)$ & $108(92)$ \\
\hline & & 60 & & $115(82)$ & $105(82)$ & $105(89)$ & $100(92)$ \\
\hline & & 90 & & $125(85)$ & $112(85)$ & $110(89)$ & $112(92)$ \\
\hline & & 120 & & $135(88)$ & $115(86)$ & $115(91)$ & $115(92)$ \\
\hline
\end{tabular}

[\% Retention of sorbates from calculated values in parenthesis]. 
equal combined carbonized and uncarbonized goat hair from experimental and calculated values at different particles size and contact time. Using $325 \mu \mathrm{m}$, crude oil was retained most at $120 \mathrm{~min}\left(1^{\text {st }}\right.$ and $\left.2^{\text {nd }}\right)$ use (experiment). While calculated occurred highest at 120 and $60 \mathrm{~min}$ at the same use. Diesel was retained highest at 90 and 120 mins $\left(1^{\text {st }}\right.$ and $\left.2^{\text {nd }}\right)$ use, considering experimental value while calculated retained highest at 120 mins for both uses. Kerosene was retained most at 120 mins for all applications during the experimental, calculated value followed the same trend but had minor variation. The same was observed for petrol. Considering $625 \mu \mathrm{m}$, crude oil was retained highest at 120 and 60 - 90 mins contact time for the experimental at $1^{\text {st }}$ and $2^{\text {nd }}$ use, calculated retention was highest at $120 \mathrm{mins}$ at the same application. Diesel retention values for both experiment and calculated were highest at 120 mins contact time for all applications. The same was observed for kerosene and petrol but for minor variations.

Table 7: Percentage retention profiles of sorbates by combined carbonized and uncarbonized coco-nut husk from experimental and calculated values at different particle size and contact time. Considering retention by 325 $\mu \mathrm{m}$ particles size and except for minor variations, crude oil was retained highest at 120 mins by both experimental and calculation at all usage. The same trend was observed for diesel, kerosene and petrol. Using $625 \mu \mathrm{m}$ and for crude oil, highest retention from experiment was at 120 mins during $1^{\text {st }}$ and $2^{\text {nd }}$ use, while that calculation was at 90 mins for the same application, for diesel (experiment) was retained most at 60 and 90 mins, that of calculated were at 120 mins. Kerosene was retained highest at 60 and 120 mins for $1^{\text {st }}$ and $2^{\text {nd }}$ to $4^{\text {th }}$ use considering experimental values while calculated values were highest at 120 mins. Petrol was retained highest at 60,90 and 120 mins during $2^{\text {nd }}$ use for experimental consideration but values had highest retention at $120 \mathrm{~min}$ for $1^{\text {st }}$ and $2^{\text {nd }}$ application.

Table 8: Percentage retention profiles of sorbates by combines carbonized and uncarbonized corn-cob from experimental and calculated values at different particle size and contact time, using $325 \mu \mathrm{m}$, crude oil was retained highest at 120 and 90 mins time at $1^{\text {st }}$ and $2^{\text {nd }}$ use (experiment) while that of calculated were retained most at $90 \mathrm{~min}$ for both $1^{\text {st }}$ and $2^{\text {nd }}$ application, that of diesel were at 120 and $90 \mathrm{~min}, 90$ and $60 \mathrm{mins}$ for calculated value. For experimental and calculated retention, that of kerosene occurred highest at $120 \mathrm{~min}$ for $1^{\text {st }}$ and $2^{\text {nd }}$ use petrol was retained highest at $120 \mathrm{~min}\left(1^{\text {st }}\right.$ use $)$ for experiment while calculated was 30 mins. Using $325 \mu \mathrm{m}$, highest retention of crude oil occurred at 90 and 120 mins while calculated values were highest at 30 and $90 \mathrm{mins}$ at $1^{\text {st }}$ and $2^{\text {nd }}$ use. Diesel was retained highest during $1^{\text {st }}$ and $2^{\text {nd }}$ application at 90 mins (experimental) while calculated was at 120 and $60 \mathrm{~min}$. at 90 and 120 min contact time, experimental value of kerosene was highest at $1^{\text {st }}$ and $2^{\text {nd }}$ use that of calculated was at 120 mins for both use, that of petrol occurred at $120 \mathrm{~min}$ and at $30 \mathrm{~min}$ for experimental and calculated value during $1^{\text {st }}$ and $2^{\text {nd }}$ use.

Table 9: \% protein of feather were higher than that of goat hair at all particle size and treatment, at $325 \mu \mathrm{m}$ carbonized feather has higher protein content than goat hair, at $625 \mu \mathrm{m}$ the same was observed (but very slightly) for the uncarbonized at $325 \mu \mathrm{m}$, feather had a slightly higher protein than goat hair. At $625 \mu \mathrm{m}$, the same trend was observed.

Table 10: A striking observation was noticed in table 10 , in which it was noticed that for both carbonized and uncarbonized, smaller particle size $(325 \mu \mathrm{m})$ contained less cellulose (in g) than large particle size $(625 \mu \mathrm{m})$ for both coconut husk and corn cob. The reverse was observed in \% lignin content. It was observed that smaller particle size $(325 \mu \mathrm{m})$ of the same sorbents (coco-nut husk and corn cob), either carbonized or uncarbonized had higher percentage lignin content than larger particle size $(625 \mu \mathrm{m})$.

Table 11: Shows that all the sorbents contained all the heavy metals analyzed. $\mathrm{Fe}^{2+}$ was more in abundant, followed by $\mathrm{Cr}^{3+}$, then $\mathrm{Mn}^{2+}$ and $\mathrm{Ni}^{2+}$ least (although minor variation) occurred. For carbonized feather, $325 \mu \mathrm{m}$ had the least of the metal ions while $625 \mu \mathrm{m}$ had highest $\mathrm{Cr}^{3+}$, $\mathrm{Fe}^{2+}, \mathrm{Ni}^{2+}$ and $\mathrm{Mn}^{2+}$. For the uncarbonized feather, 325 $\mu \mathrm{m}$ had highest $\mathrm{Cr}^{3+}$ and $\mathrm{Fe}^{2+}$ while $625 \mu \mathrm{m}$ had highest $\mathrm{Ni}^{2+}$ and $\mathrm{Mn}^{2+}$.

For the goat hair, carbonized $325 \mu \mathrm{m}$ had least of all the metals while $625 \mu \mathrm{m}$ had the highest. For the uncarbonized, $325 \mu \mathrm{m}$ had highest $\mathrm{Fe}^{2+}$ while $625 \mu \mathrm{m}$ had highest $\mathrm{Cr}^{3+}$ and $\mathrm{Mn}^{2+}$, both had equal $\mathrm{Ni}^{2+}$.

For coco-nut husk, carbonized $625 \mu \mathrm{m}$ had highest values of all the metals analyzed than $325 \mu \mathrm{m}$, for the uncarbonized, $325 \mu \mathrm{m}$ had highest $\mathrm{Ni}^{2+}$ while $625 \mu \mathrm{m}$ had highest $\mathrm{Cr}^{3+}, \mathrm{Fe}^{2+}$ and $\mathrm{Mn}^{2+}$.

For corn cob, carbonized $625 \mu \mathrm{m}$ had the highest values for all the metals $\left(\mathrm{Cr}^{3+}, \mathrm{Fe}^{2+}, \mathrm{Ni}^{2+}\right.$ and $\left.\mathrm{Mn}^{2+}\right)$ while $325 \mu \mathrm{m}$ had the least. For the uncarbonized $325 \mu \mathrm{m}$ had highest $\mathrm{Ni}^{2+}$ while $625 \mu \mathrm{m}$ had highest $\mathrm{Cr}^{3+}, \mathrm{Fe}^{2+}$ and $\mathrm{Mn}^{2+}$.

Generally, $625 \mu \mathrm{m}$ of either carbonized or uncarbonized for all the sorbents contained highest values of all the metal ions $\left(\mathrm{Cr}^{3+}, \mathrm{Fe}^{2+}, \mathrm{Ni}^{2+}\right.$ and $\left.\mathrm{Mn}^{2+}\right)$ but for few exceptions.

\section{Discussion}

The results of the present study showed high percentage absorption/adsorption, recovery and retention of all the four liquids (sorbates) by the sorbents both carbonized 
Table 7. Percentage retention profiles of sorbates by combined carbonized and uncarbonized coco-nut husk from experimental and calculated values at different particles size and contact time.

\begin{tabular}{|c|c|c|c|c|c|c|c|}
\hline \multicolumn{8}{|c|}{$\%$ Retention } \\
\hline S/No & Particle size & Contact time (min) & Sorbates & $1^{\text {st }}$ Use & $2^{\text {nd }}$ Use & $3^{\text {rd }}$ Use & $4^{\text {th }}$ Use \\
\hline \multirow{4}{*}{1} & \multirow{4}{*}{$325 \mu \mathrm{m}$} & 30 & \multirow{4}{*}{ Crude Oil } & $260(106)$ & $200(173)$ & $202(161)$ & $200(113)$ \\
\hline & & 60 & & $280(127)$ & $200(178)$ & $190(168)$ & $205(111)$ \\
\hline & & 90 & & $310(122)$ & $260(159)$ & $200(166)$ & $210(114)$ \\
\hline & & 120 & & $325(128)$ & $200(158)$ & $195(161)$ & $210(121)$ \\
\hline \multirow{4}{*}{2} & \multirow{4}{*}{$325 \mu \mathrm{m}$} & 30 & \multirow{4}{*}{ Diesel } & $180(154)$ & $200(1180$ & $200(131)$ & $190(129)$ \\
\hline & & 60 & & $200(170)$ & $210(124)$ & $220(133)$ & $190(130)$ \\
\hline & & 90 & & $250(179)$ & $200(132)$ & $210(135)$ & $200(126)$ \\
\hline & & 120 & & $240(182)$ & $215(128)$ & $200(141)$ & $205(130)$ \\
\hline \multirow{4}{*}{3} & \multirow{4}{*}{$325 \mu \mathrm{m}$} & 30 & \multirow{4}{*}{ Kerosene } & $140(114)$ & $110(116)$ & $110(116)$ & $106(111)$ \\
\hline & & 60 & & $170(117)$ & $125(119)$ & $115(119)$ & $110(112)$ \\
\hline & & 90 & & $165(115)$ & $140(118)$ & $110(118)$ & $110(116)$ \\
\hline & & 120 & & $175(113)$ & 144 (119) & $140(117)$ & $105(120)$ \\
\hline \multirow{4}{*}{4} & \multirow{4}{*}{$325 \mu \mathrm{m}$} & 30 & \multirow{4}{*}{ Petrol } & $110(106)$ & $110(106)$ & $104(110)$ & $90(115)$ \\
\hline & & 60 & & $120(110)$ & $110(107)$ & $95(113)$ & $105(118)$ \\
\hline & & 90 & & $133(104)$ & $110(107)$ & $100(111)$ & $115(114)$ \\
\hline & & 120 & & $155(100)$ & $150(110)$ & $122(112)$ & 115 (114) \\
\hline \multirow{4}{*}{5} & \multirow{4}{*}{$625 \mu \mathrm{m}$} & 30 & \multirow{4}{*}{ Crude Oil } & $190(139)$ & $125(135)$ & $200(112)$ & $178(100)$ \\
\hline & & 60 & & $215(145)$ & $160(138)$ & $210(112)$ & $183(106)$ \\
\hline & & 90 & & $205(146)$ & $185(141)$ & $210(115)$ & $195(102)$ \\
\hline & & 120 & & $320(142)$ & $185(138)$ & $140(115)$ & $190(108)$ \\
\hline \multirow{4}{*}{6} & \multirow{4}{*}{$625 \mu \mathrm{m}$} & 30 & \multirow{4}{*}{ Diesel } & $200(115)$ & $170(103)$ & $155(102)$ & $143(114)$ \\
\hline & & 60 & & $211(116)$ & $170(104)$ & $160(104)$ & 145 (113) \\
\hline & & 90 & & $145(116)$ & $185(104)$ & $210(103)$ & 185 (117) \\
\hline & & 120 & & $165(119)$ & $150(105)$ & $210(105)$ & 195 (117) \\
\hline \multirow{4}{*}{7} & \multirow{4}{*}{$625 \mu \mathrm{m}$} & 30 & \multirow{4}{*}{ Kerosene } & $129(98)$ & $118(102)$ & $120(76)$ & $112(93)$ \\
\hline & & 60 & & 130 (99) & $120(107)$ & $119(76)$ & $114(95)$ \\
\hline & & 90 & & $128(105)$ & 120 (109) & $120(78)$ & $115(92)$ \\
\hline & & 120 & & $125(107)$ & $130(106)$ & $125(78)$ & $115(94)$ \\
\hline \multirow{4}{*}{8} & \multirow{4}{*}{$625 \mu \mathrm{m}$} & 30 & \multirow{4}{*}{ Petrol } & $104(86)$ & $100(81)$ & $139(84)$ & $101(82)$ \\
\hline & & 60 & & $120(88)$ & $110(81)$ & $110(87)$ & $100(83)$ \\
\hline & & 90 & & $120(88)$ & $115(82)$ & $120(88)$ & $110(83)$ \\
\hline & & 120 & & $115(90)$ & $120(83)$ & $115(87)$ & $105(84)$ \\
\hline
\end{tabular}

[\% Retention of sorbates from calculated values in parenthesis]. 
Table 8. Percentage retention profiles of sorbates by combined carbonized and uncarbonized corn cob from experimental and calculated values at different particles size and contact time.

\begin{tabular}{|c|c|c|c|c|c|c|c|}
\hline \multicolumn{8}{|c|}{$\%$ Retention } \\
\hline S/No & Particle size & Contact time (min) & Sorbates & $1^{\text {st }}$ Use & $2^{\text {nd }}$ Use & $3^{\text {rd }}$ Use & $4^{\text {th }}$ Use \\
\hline \multirow{4}{*}{1} & \multirow{4}{*}{$325 \mu \mathrm{m}$} & 30 & \multirow{4}{*}{ Crude Oil } & $192(105)$ & $168(132)$ & $180(144)$ & $170(101)$ \\
\hline & & 60 & & $120(108)$ & $180(156)$ & $190(162)$ & $172(111)$ \\
\hline & & 90 & & $230(150)$ & $192(151)$ & $150(146)$ & $180(129)$ \\
\hline & & 120 & & $285(130)$ & $158(142)$ & $140(145)$ & $174(104)$ \\
\hline \multirow{4}{*}{2} & \multirow{4}{*}{$325 \mu \mathrm{m}$} & 30 & \multirow{4}{*}{ Diesel } & $166(85)$ & $135(78)$ & $125(92)$ & $168(96)$ \\
\hline & & 60 & & $160(142)$ & $153(148)$ & $160(109)$ & $180(85)$ \\
\hline & & 90 & & $154(152)$ & $187(142)$ & $172(109)$ & $182(78)$ \\
\hline & & 120 & & $274(95)$ & $178(130)$ & $160(103)$ & $167(77)$ \\
\hline \multirow{4}{*}{3} & \multirow{4}{*}{$325 \mu \mathrm{m}$} & 30 & \multirow{4}{*}{ Kerosene } & 104 (109) & $102(123)$ & $84(164)$ & $104(154)$ \\
\hline & & 60 & & $106(115)$ & $98(134)$ & $88(167)$ & $124(164)$ \\
\hline & & 90 & & $94(126)$ & $96(133)$ & $90(127)$ & $110(152)$ \\
\hline & & 120 & & $120(139)$ & $128(129)$ & $128(147)$ & $140(141)$ \\
\hline \multirow{4}{*}{4} & \multirow{4}{*}{$325 \mu \mathrm{m}$} & 30 & \multirow{4}{*}{ Petrol } & $106(103)$ & $85(124)$ & $97(93)$ & $100(75)$ \\
\hline & & 60 & & $90(102)$ & $85(132)$ & $90(105)$ & $110(77)$ \\
\hline & & 90 & & $90(99)$ & $75(122)$ & $95(105)$ & $105(56)$ \\
\hline & & 120 & & 125 (97) & $50(116)$ & $100(105)$ & $125(71)$ \\
\hline \multirow{4}{*}{5} & \multirow{4}{*}{$625 \mu \mathrm{m}$} & 30 & \multirow{4}{*}{ Crude Oil } & $160(110)$ & $120(89)$ & $220(83)$ & $210(63)$ \\
\hline & & 60 & & $200(95)$ & $170(86)$ & $213(87)$ & $220(75)$ \\
\hline & & 90 & & $195(108)$ & $172(99)$ & $220(86)$ & $220(73)$ \\
\hline & & 120 & & $280(107)$ & $102(79)$ & $220(88)$ & $220(77)$ \\
\hline \multirow{4}{*}{6} & \multirow{4}{*}{$625 \mu \mathrm{m}$} & 30 & \multirow{4}{*}{ Diesel } & $152(98)$ & $156(115)$ & $130(60)$ & $140(59)$ \\
\hline & & 60 & & $160(100)$ & $162(117)$ & $139(60)$ & $139(55)$ \\
\hline & & 90 & & 202 (99) & $170(103)$ & $192(58)$ & $196(57)$ \\
\hline & & 120 & & $160(104)$ & $146(102)$ & $198(61)$ & $190(52)$ \\
\hline \multirow{4}{*}{7} & \multirow{4}{*}{$625 \mu \mathrm{m}$} & 30 & \multirow{4}{*}{ Kerosene } & $122(87)$ & $120(97)$ & $120(88)$ & $106(85)$ \\
\hline & & 60 & & $125(102)$ & $116(99)$ & $116(73)$ & $110(85)$ \\
\hline & & 90 & & $126(99)$ & $115(94)$ & $120(77)$ & $110(82)$ \\
\hline & & 120 & & $120(104)$ & $118(103)$ & $122(80)$ & $110(85)$ \\
\hline \multirow{4}{*}{8} & \multirow{4}{*}{$625 \mu \mathrm{m}$} & 30 & \multirow{4}{*}{ Petrol } & $102(104)$ & $102(71)$ & $102(18)$ & $94(23)$ \\
\hline & & 60 & & 114 (97) & $106(66)$ & $104(35)$ & $100(19)$ \\
\hline & & 90 & & 114 (109) & $110(66)$ & $104(32)$ & $108(20)$ \\
\hline & & 120 & & $120(97)$ & $123(64)$ & $112(36)$ & $112(21)$ \\
\hline
\end{tabular}

[\% Retention of sorbates from calculated values in parenthesis]. 
Table 9. \% protein content of feather and goat hair.

\begin{tabular}{cccc}
\hline S/No & Sorbent nature & Particle size & \% Protein \\
\hline 1 & Carbonized feather & $325 \mu \mathrm{m}$ & 4.25 \\
2 & Carbonized feather & $625 \mu \mathrm{m}$ & 4.45 \\
3 & Uncarbonized feather & $325 \mu \mathrm{m}$ & 4.33 \\
4 & Uncarbonized feather & $625 \mu \mathrm{m}$ & 4.38 \\
5 & Carbonized goat hair & $325 \mu \mathrm{m}$ & 3.85 \\
6 & Carbonized goat hair & $625 \mu \mathrm{m}$ & 4.40 \\
7 & Uncarbonized goat hair & $325 \mu \mathrm{m}$ & 4.38 \\
8 & Uncarbonized goat hair & $625 \mu \mathrm{m}$ & 4.55 \\
\hline
\end{tabular}

Table 10. Cellulose and lignin content of coco-nut husk (coir) and corn cob hair.

\begin{tabular}{ccccc}
\hline S/No & Sorbent nature & Particle size & Cellulose content (g) & \% Lignin content \\
\hline 1 & Carbonized coco-nut husk & $325 \mu \mathrm{m}$ & 3.22 & 70.80 \\
2 & Carbonized coco-nut husk & $625 \mu \mathrm{m}$ & 7.42 & 49.60 \\
3 & Uncarbonized coco-nut husk & $325 \mu \mathrm{m}$ & 4.53 & 80.10 \\
4 & Uncarbonized coco-nut husk & $625 \mu \mathrm{m}$ & 8.24 & 59.20 \\
5 & Carbonized corn cob & $325 \mu \mathrm{m}$ & 6.19 & 60.00 \\
6 & Carbonized corn cob & $625 \mu \mathrm{m}$ & 9.35 & 38.90 \\
7 & Uncarbonized corn cob & $325 \mu \mathrm{m}$ & 2.55 & 69.80 \\
8 & Uncarbonized corn cob & $625 \mu \mathrm{m}$ & 2.94 & 40.90 \\
\hline
\end{tabular}

Table 11. Heavy content of the proteinous and cellulosic sorbents (both carbonized and (metal) uncarbonized).

\begin{tabular}{|c|c|c|c|c|c|c|}
\hline \multirow{2}{*}{ S/No } & \multirow{2}{*}{ Sorbent nature } & \multirow{2}{*}{ Particle size } & \multicolumn{4}{|c|}{ Heavy metal content in $\mathrm{mg} / \mathrm{kg}$} \\
\hline & & & $\mathrm{Cr}^{3+}$ & $\mathrm{Fe}^{2+}$ & $\mathrm{Ni}^{2+}$ & $\mathrm{Mn}^{2+}$ \\
\hline 1 & Carbonized feather & $325 \mu \mathrm{m}$ & 0.95 & 2.00 & 0.18 & 0.64 \\
\hline 2 & Carbonized feather & $625 \mu \mathrm{m}$ & 1.44 & 2.66 & 0.33 & 1.08 \\
\hline 3 & Uncarbonized feather & $325 \mu \mathrm{m}$ & 2.42 & 10.00 & 0.15 & 1.08 \\
\hline 4 & Uncarbonized feather & $625 \mu \mathrm{m}$ & 0.87 & 7.33 & 0.25 & 1.43 \\
\hline 5 & Carbonized goat hair & $325 \mu \mathrm{m}$ & 0.29 & 4.83 & 0.53 & 1.05 \\
\hline 6 & Carbonized goat hair & $625 \mu \mathrm{m}$ & 0.99 & 5.08 & 0.62 & 1.32 \\
\hline 7 & Uncarbonized goat hair & $325 \mu \mathrm{m}$ & 2.46 & 4.88 & 0.10 & 1.04 \\
\hline 8 & Uncarbonized goat hair & $625 \mu \mathrm{m}$ & 2.84 & 4.66 & 0.10 & 2.52 \\
\hline 9 & Carbonized coco-nut husk & $325 \mu \mathrm{m}$ & 2.52 & 5.16 & 0.08 & 0.62 \\
\hline 10 & Carbonized coco-nut husk & $625 \mu \mathrm{m}$ & 2.89 & 9.00 & 0.19 & 1.64 \\
\hline 11 & Uncarbonized coco-nut husk & $325 \mu \mathrm{m}$ & 1.63 & 11.66 & 0.12 & 1.17 \\
\hline 12 & Uncarbonized coco-nut husk & $625 \mu \mathrm{m}$ & 1.75 & 14.74 & 0.10 & 1.89 \\
\hline 13 & Carbonized corn cob & $325 \mu \mathrm{m}$ & 0.22 & 1.50 & 0.05 & 0.80 \\
\hline 14 & Carbonized corn cob & $625 \mu \mathrm{m}$ & 0.92 & 3.33 & 0.33 & 0.98 \\
\hline 15 & Uncarbonized corn cob & $325 \mu \mathrm{m}$ & 0.56 & 8.08 & 0.92 & 0.73 \\
\hline 16 & Uncarbonized corn cob & $625 \mu \mathrm{m}$ & 1.64 & 13.83 & 0.07 & 1.52 \\
\hline
\end{tabular}


and uncarbonized and in comparing experimental with calculated values (Tables 1(a)-4(a), 1(b)-4(b) and 5-8). A closer look at the tables revealed that percentage absorption/adsorption of sorbates by sorbents were high in experiment than calculated value, but when considering percentage recovery, it was observed that variations do exist, in some cases percentage recovery of calculated value being higher than that of experimental values (Tables 1(a)-4(a) and 1(b)-4(b)), this has to do with the mechanism of absorption/adsorption which will be explained later; the same influences the high percentage retention observed in experimental than calculated value (Tables 5-7), except that of corn cob (Table 8). Experimental values were obtained by equal combination of the same grammes $(0.75 \mathrm{~g}$ each of both carbonized and uncarbonized sorbents of the same particle size and used in mopping up of the hydrocarbon liquids while calculated values were obtained by using individual sorbents either carbonized or uncarbonized to mop-up the hydrocarbons, their average results reported as calculated value (i.e. percentage absorption/adsorption of carbonized sorbent plus percentage absorption/adsorption of uncarbonized sorbent of the same particle size divided by two (2) is given as calculated percentage absorption; percentage recovery of carbonized sorbent plus percentage recovery of uncarbonized sorbent of the same particle size divided by two (2) is given as calculated percentage recovery; percentage retention of carbonized sorbents plus percentage retention of uncarbonized sorbents of the same particle size divided by two (2) is given as calculated percentage retention (Tables 1(a)-4(b)), all these were obtained from a previous manuscript [12] and then compared with experimental results (Tables 1(a)-4(a)).

The results are very explainable, the sorbents mopped up appreciable quantities of the hydrocarbon liquids often more than $500 \%$ of their weights of the hydrocarbon sorbates within an average of 90 mins of contact time. Large volumes of the sorbates were equally recovered by mere hydraulic pressing. What is clearly evident by inspection is that the nature of hydrocarbon liquids (sorbates) has overriding influence on the relative affinities of the sorbents for the sorbates. Although the sorbents can be re-used several times [16], this study stopped at $4^{\text {th }}$ re-use, it was observed that though there was high percentage absorption/adsorption, recovery and retention at each use, a gradual decrease across the table was observed. Also the longer the contact time, the more the hydrocarbon absorbed/adsorbed, recovered and retained, hence more of the hydrocarbons (sorbates) were sorbed, recovered and retained at $120 \mathrm{~min}$ contact time for every application (though minor variations exist). The reason why experimental values were higher than calculated values may be attributed to mechanism of absorption/adsorption inherent in the study. Sorbents can cap- ture oil by three mechanisms: 1) adsorption to the surface of the sorbents; 2) absorption into spaces among aggregated granules of fibres of sorbent (primary absorption) and 3) absorption into spaces within individual granules or fibres of sorbents (pores and capillaries), it is termed secondary absorption [16]. All these were present in our present study as against previous study from which calculated values were obtained, where only two mechanisms were involved - adsorption to the surface of the sorbent and primary absorption hence the calculated values were less [12], when compared with the experimental results. This is so because in the work [12] from which we got our calculated values, adsorption was on the surface and into the pores and capillaries of individual and between two particles (voids) of a given sorbent whether carbonized or uncarbonized taken singly but in the present study adsorption is on the surface of both carbonized and uncarbonized sorbents, then absorption into the pores and capillaries of both carbonized and uncarbonized, absorption into the voids between two or more particles of uncarbonized sorbents and finally absorption into voids between two or more particles of both carbonized and uncarbonized sorbents. The irregular shapes of the particles and in-homogeneities in the packing of the particles created more voids for absorption of sorbates, hence an advantage for combining the sorbents.

This invariably account for the high percentage retention because during recovery by hydraulic pressing, sorbates on the surface and some among aggregated granules are easily desorbed while some among the same aggregated granules and those absorbed into spaces (pores and capillaries) within individual granules are retained. Another reason is that during carbonization, a more complex and solid structure is formed, because pores increase in number and size, new ones appear, and two or more pores can merge into one with more pore surface and volume [17]. This shows that the sorbents in this study are good for oil/chemical spill clean-up because a good sorbent must have high degree of absorption and retention [16]. A number of natural features inherent in the sorbents which was ignored in the previous report [11] make them to have high sorption capacity for oil and oil products. Previous study has shown that cotton with enormous surface having fibril/fibers absorbed more than twice the volume of these hydrocarbon liquids than materials of this study [18]. Coco-nut husk and corn-cob are both fibrous and granular (having been ground) will have good primary absorption characteristics (absorption into air - spaces among aggregated granules or fibers of sorbents), [19-21]. Also both being plants, have hollow internal conduits; xylem for transporting water and phloem for transporting dissolved nutrients and therefore can be good for secondary absorption (absorption into pits, lacunae or lumina, within indi- 
vidual granules or fibres of sorbents); therefore it is evident that both primary and secondary absorption has overriding influence in sorption capacity of uncarbonized cellulosic sorbents. Uncarbonized protein sorbents (feathers and goat hair) have a natural waxy, water-proof coatings which makes oleophilicity possible. Modification (activation or carbonization) may enhance the sorption capacities of the sorbents. Moderate heat treatment of cellulosic particles/fibres improves their sorbency because it causes the production of oleophilic resins [22]. Heat treatment of cellulosic sorbents (coir and corn cob) as was done here, altered their chemical composition to make it more hydrophobic and, hence more oleophilic. It is established that pyrolysis (heat activation) at $200^{\circ} \mathrm{C}$ $500^{\circ} \mathrm{C}$ improves their hydrophobic and oleophilic properties [22]. Although treatment improves the oil sorption capacity of sorbents, moderate treatment as was done here is recommended because excess refinement might strip surface chemicals that are important for the adsorption of oil [16].

Modification (activation), heating and chemical treatment (as was done in this study) increases the sorbents affinity to oil and repellency to water by enhancing their structural integrity, the weak bonds associated with oxygen in the lignin and cellulose structure are easily broken. Cellulose is a naturally occurring long chain polymer containing a series of glycosodic rings joined by oxygen bridges [23], while lignins are polyphenolic cell wall constituents connected to the polysaccharide constituent by chemical bonds [24]. Heat and chemical treatment also drives out water and volatile matter leading to more vacant sites for oil absorption and retention in internal spaces and their interstices (Figures 1 and 2). In both carbonized and uncarbonized, smaller particle size (325 $\mu \mathrm{m})$ contained less cellulose than large particle $(625 \mu \mathrm{m})$ in both coco-nut husk and corn cob, the same was also observed if considering lignin (Table 10) and since smaller particle size had more percentage absorption/ adsorption, it therefore implies that size reduction, packing and destruction of plant constituent enhances oil/ chemical sorption. Therefore secondary absorption has overriding effect in activated sorbents while primary absorption dominates in unactivated protein sorbents because of their long fibres and large external surface area and surface-coatings that are oleophilic. It has been reported [25] that one (1) gramme of feathers has a greater surface area than any known sorbent materials but must exclude cotton [18]. It was also observed that proteinous sorbents with smaller particle size $(325 \mu \mathrm{m})$, that is large surface contains lesser protein than large particle size (625 $\mu \mathrm{m})$, whether carbonized or uncarbonized (Table 9). Since smaller particle size absorb more hydrocarbon liquid than large particle size, it means that protein content is not overriding but natural constituents enhanced by mild treatment, but protein content is an important factor because at $325 \mu \mathrm{m}$ and $625 \mu \mathrm{m}$ carbonized feather has higher protein than goat hair. Since it is established that protein sorbent has natural chemical coating with great affinity for oil [21]. The presence of heavy metals (Table 11) in the sorbents is not surprising, as it is an established fact that organic compounds (which the sorbents are), are associated with heavy metals [26-29]. The sorbents were deliberately examined for some of the metals $\left(\mathrm{Cr}^{3+}, \mathrm{Fe}^{2+}\right.$, $\mathrm{Ni}^{2+}$ and $\mathrm{Mn}^{2+}$ ) [30], which have vacant d-orbitals into which the hydrocarbons liquid may be absorbed. The metals may not occur in Free State but as transition metal ion complexes with four (4) to six (6) ligands surrounding the central ion. The bonding between the ligands and

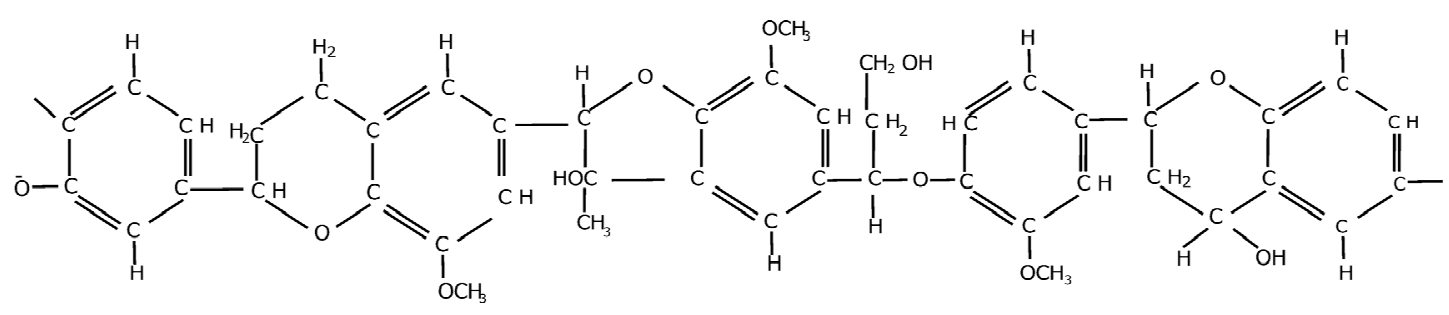

Figure 1. Possible structure for lignin monomer accounting for some of its properties.

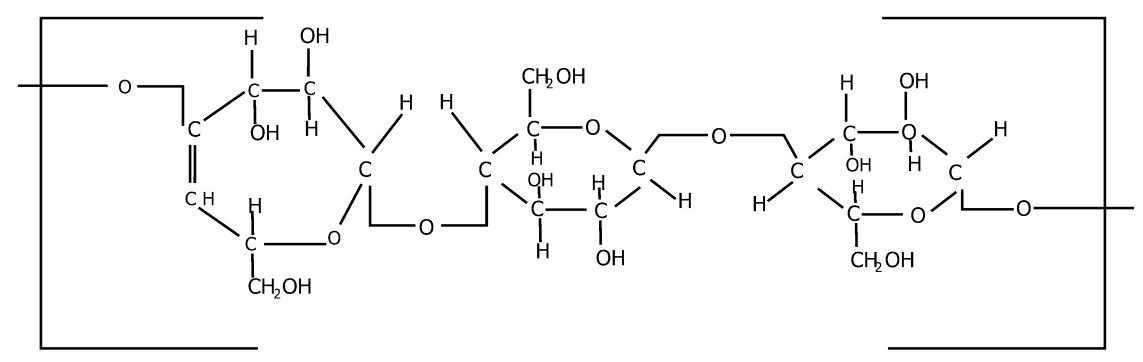

Figure 2. Structure formula of cellulose. 
the metal ion can be ionic or covalent or intermediate between the two [30]. Chemical and heat treatment (activation) as was done here decomposes the metal ion complex to create sites for absorption, this may partly contribute to high sorption capacity of activated sorbents. Also except for the uncarbonized feather, $325 \mu \mathrm{m}$ contained less of all the metals than $625 \mu \mathrm{m}$ in both carbonized and uncarbonized sorbents meaning that some of the metals must have escaped with the volatile matter during carbonization and hence more vacant site for oil sorption. Surveying the results again (Tables 1(a)-4(a), 1(b)-4(b) and 5-8) mirrors on already established trend; in which the hydrocarbon liquids were sorbed in the order; crude oil $>$ diesel $>$ kerosene $>$ petrol. Molecular weight (chain length) of sorbates, viscosities and structure are the dominant factors while sorbent features such as natural che$\mathrm{mical} / \mathrm{physical}$ constituents, pores/voids, convolutions, lumen/lumina, lacunae, inter-fibril/fiber spaces, chemicals released by activation, specific adsorption/absorption sites improved by modification and combination are overriding. Since no type of chemical interactions can be attributed to the sorption of the sorbates by sorbents, therefore the binding of the hydrocarbons by the sorbents must be by physical adhesive forces such as Van der waals, london forces and other dispersion phenomena as well as physical entanglement/occlusion. These are nonbonding interactions that are important in several fields of chemistry. Going back to the results again (Tables 1(a)-4(a) and 1(b)-4(b)), it is observed that there is reduction in the quantity of sorbents adsorbed/absorbed and recovered on re-use (across the tables), this may be because of saturation of pores and capillaries of the sorbents, hence high retention (Tables 5-8). The variations observed throughout the tables may be due to the fact that absorption/adsorption-desorption phenomena is nonstochiometry. Further more since the investigations were carried out at ambient temperature and pressure, the effect of these external influences can be considered uniform. Atmospheric features (wind and humidity) and environmental factors (noise and structural vibration) are important factors. It can also be attributed to impurities in sorbents and sorbates. High volatility of the sorbates even at ambient temperatures can be considered as an important factor. From this work, excluding minor variation, it is established that protein sorbents have high percentage absorption capacity than cellulosic sorbents but the later tend to be more abundant and therefore cost effective but both can therefore serve as alternative to synthetic polyurethane foam already in use by oil industry operators and oil spill clean-up organizations, hence an economical utilization of waste. The sorbents (feather, goat, coco-nut husk and corn cob) has also been found to be highly efficient in detoxification of waste water [31]. These applications are effective means of ridden the en- vironment of waste and ensure environmental cleanliness and improve public health. Leachability test of the sorbents conducted with kerosene to ascertain percentage release of the hydrocarbon into the environment, it shows that sorbents with highest sorption capability, leached the least sorbates and vice versa, the leaching order is thuscorn cob $>$ coco-nut husk $>$ goat hair $>$ feather (Figure 3). If the sorbents, after use were left in an open dump and impacted by rain, it was observed that comparing leachability tests of crude oil [11], diesel [12] and that of kerosene reported here (Figure 3), it was evident that the low molecular weight of the three had the greatest release but was below $5 \%$, so they do not constitute serious environmental menace if left in a open dump to decay since being cellulosic or proteinous they are biodegradable, they can also be burnt.

\section{Conclusion}

It is concluded that combining carbonized and uncarbonized sorbents of the same particle size absorbed the four hydrocarbon liquids more than any single use. That carbonization and particle size of the sorbents as well as chain length and viscosity of the sorbates are major determinant factors. Natural inherent features and modify-

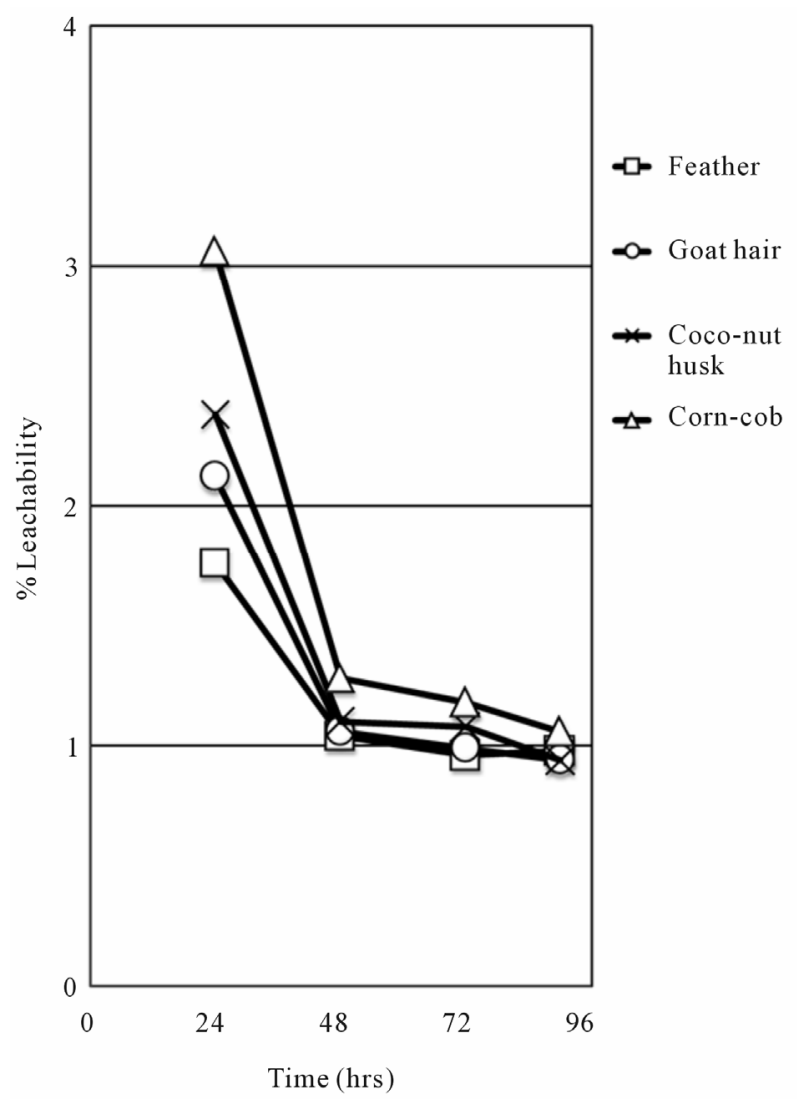

Figure 3. Percentage leachability of residual kerosene by the sorbents without regard to activation or particle size. 
cation of the sorbents contributed to their sorption capacities. High percentage adsorption/absorption, recovery and retention were recorded. The sorbents have very small percentage leachability. Three sorption mechanism types (adsorption on the surface, primary and secondary absorption) were involved.

\section{REFERENCES}

[1] O. T. Adeyemi, "Oil Exploration and Environmental Degradation: The Nigerian Experience," Environmental Informatics Archives, Vol. 2, 2004, pp. 387-393.

[2] T. J. A. Reijers, S. W. Petters and C. S. Nwajide, "The Niger Delta Basin. In Selected Chapters on Geology, Sedimentary Geology and Stratigraphy in Nigeria," SPDC, Nigeria, 1996, pp. 103-117.

[3] SPDC, "Oil/Chemical Spill Contingency Plan and Procedures," Bulletin, Nigeria, 1997.

[4] A. Birk, "Chemistry," Houghton Mifflin Company, New York, 1994, pp. 980-981.

[5] A. Chem, "Effect of Mixing Conditions on Flocculation Kinetics of Waste Water Containing Using Fibrous Materials and Polyelectrolytes," Brazilian Journal of Chemical Engineering, Vol. 15, 1998, p. 350.

[6] Minnesota Mining \& Manufacturing, "Method of Making Sorbent Article,” European Patent No. EP0608884, 1994.

[7] D. Ronald, "Technical Applications for Wool Helps Improve the Environment," Technical Textile International, Vol. 2, 1993, p. 8.

[8] A. Kugin, "Obtaining of High Effective Absorbents on the Basis of Wood Fibrous Materials," Forest Chemistry and Organic Synthesis, Vol. 10, No. 5, 1994, p. 3.

[9] S. Nenkova, "Study of Sorption Properties of LigninDerivatized Fibrous Composites for the Remediation of Oil Polluted Receiving Waters," BioResources, Vol. 2, No. 3, 2007, pp. 408-418.

[10] L. Grigorieve, "Obtaining and Application of New Materials on the Basis of Lignin Waste," Cellulose, Vol. 3, 1996, p. 24.

[11] J. K. Nduka, L. O. Ezenweke and T. E. Ezenwa, "Comparison of the Mopping Ability of Chemically Modified and Unmodified Biological Wastes on Crude Oil and Its Lower Fraction," BioResource Technology, Vol. 99, No. 16, 2008, pp. 7902-7905. doi:10.1016/j.biortech.2008.01.066

[12] J. K. Nduka and V. I. Uchegbusi, "Extension of Comparison of the Mopping Ability of Chemically Modified and Unmodified Biological Wastes on Crude Oil and Its Lower Fractions," British Journal of Environment and Climate Change, 2012, in press.

[13] M. B. Jacobs, "Chemical Analysis of Food and Products," Van Nostrand Company Inc., New York, 1938.

[14] D. M. Updegraff, "Semi-Micro Determination of Cellulose in Biological Materials," Analytical Biochemistry, Vol. 32, 1969, pp. 420-424. doi:10.1016/S0003-2697(69)80009-6

[15] C. 1. Aquiar, "Biodegradation of the Cellulose from Sug- arcane Bagasse by Fungal Cellulose," Ciência e Tecnologia de Alimentos, Vol. 3, No. 2, 2001, pp. 117-121.

[16] M. G. Hoskin, A. J. Underwood and P. Archabault, "Properties of Naturally Degrading Sorbents for Potential Use in the Clean-Up of Oil Spills in Sensitive and Remote Coastal Habitats. Centre for Research on Ecological Impacts of Coastal Cities," Marine Ecology Laboratory (All), University of Sydney NSW 2006, 2001.

[17] Z. A. Mansurov and M. K. Gilmanov, "Nano Structural Carbon Sorbents for Different Functional Application," In: T. P. Wills, Ed., Sorbents, Properties Materials and Applications, NOVA Science Publishers Inc., New York, 2009.

[18] J. K. Nduka, "The Use of Solid Wastes as Oil Spill Mop," Unpublished Master's Thesis, Nnamdi Azikiwe University, Awka, 2000.

[19] W. Widayat, P. Kusno, W. Moerdoko, W. Winiati, K. Hagiwara and P. Honde, "Research on the Development and Application of Natural Sorbents for Oil-Spill CleanUp (11). The Evaluation of Oil Absorbability of Natural Sorbents," Bulletin of the Government Industrial Research Institute, Osaka, Vol. 32, 1981, pp. 29-35.

[20] W. Winiati, W. Widayat, P. Kusno, W. Moerdoka, K. Hagiwara, K. Higashi and S. Honda, "Research on the Development and Application of Natural Sorbents for Oil-Spill Clean-Up (V): Formation and Evaluation of Mixed Sorbents Made of Kapok and Other Materials," Bulletin of the Government Industrial Research Institute, Osaka, Vol. 33, 1982, pp. 363-369.

[21] D. Cooper, S. Penton, K. Rafuse and A. B. Nordvik, "An Evaluation of Oil-Sorbent Materials," Artic and Marine Oil-Spill Program (AMOP), Technical Seminar (Vol. 1). Environment Canada, Ottawa, 1994, pp. 581-592.

[22] Y. Kato, K. Umehara and M. Aoyama, "An Oil Sorbent from Wood Fiber by Mild Pyrolysis," European Journal of Wood and Wood Products, Vol. 55, No. 6, 1997, pp. 399-401. doi:10.1007/s001070050254

[23] F. N. Emengo, J. K. C. Nduka and E. O. Onwu, "Nitration of Cellulose and Performance of Cellulose Nitrate Based Adhesives for Selected Substrates," Chemical Physics Journal, Vol. 3, No. 2-3, 2010, pp. 135-150.

[24] A. Seven and P. Rydholm, "Pulping Processes," InterScience Publishers, London, 1967.

[25] Environmental Canada, "Selection Criteria and Laboratory Evaluation of Oil-Spill Sorbents," Update 111, Ottawa, 1985.

[26] G. C. Nwandiche, "Looking for Metals in an Organic World," Unpublished Master's Thesis, Nnamdi Azikiwe University, Awka, 1998.

[27] R. W. Hay, "Bio-Inorganic Chemistry," Ellis Horwood Limited, Hemel Hempstead, 1991.

[28] C. 1. Branden, H. Jornval, H. Eklund and B. Furugren, “Alcohol Dehydrogenase," In: P. D. Boyer, Ed., The Enzyme, Vol. XI, Academic Press, New York, 1975, pp. 103-190.

[29] M. G. Simmons and I. J. Wilson, “A Hemocyanin Model: A Synthetic $\mathrm{Cu}$ (1) Complex Having Imidazole Ligands and Reversible Dioxygen Activity," Journal of the Che- 
mical Society, Chemical Communications, Vol. 15, 1978, p. 634.

[30] G. F. Liptrot, "Modern Inorganic Chemistry," 4th Edition, Unwin Hyman Ltd., London, 1983.

[31] J. K. Nduka, "Application of Chemically Modified and
Unmodified Waste Biological Sorbents in Treatment of Waste Water," International Journal of Chemical Engineering, Vol. 2012, 2012 pp. 1-7.

doi: $10.1155 / 2012 / 751240$ 